\title{
Metabolomic Insight into Polycystic Ovary Syndrome-An Overview
}

\author{
Anna Rajska ${ }^{1}$, Magdalena Buszewska-Forajta ${ }^{1}$, Dominik Rachoń ${ }^{2}$ (D) \\ and Michał Jan Markuszewski ${ }^{1, * \mathbb{D}}$ \\ 1 Department of Biopharmaceutics and Pharmacodynamics, Medical University of Gdańsk, Hallera 107, \\ 80-416 Gdańsk, Poland; anna.rajska@gumed.edu.pl (A.R.); m.buszewska-forajta@gumed.edu.pl (M.B.-F.) \\ 2 Department of Clinical and Experimental Endocrinology, Medical University of Gdańsk, Dębinki 7, \\ 80-211 Gdańsk, Poland; dominik.rachon@gumed.edu.pl \\ * Correspondence: markusz@gumed.edu.pl
}

Received: 20 May 2020; Accepted: 7 July 2020; Published: 9 July 2020

\begin{abstract}
Searching for the mechanisms of the polycystic ovary syndrome (PCOS) pathophysiology has become a crucial aspect of research performed in the last decades. However, the pathogenesis of this complex and heterogeneous endocrinopathy remains unknown. Thus, there is a need to investigate the metabolic pathways, which could be involved in the pathophysiology of PCOS and to find the metabolic markers of this disorder. The application of metabolomics gives a promising insight into the research on PCOS. It is a valuable and rapidly expanding tool, enabling the discovery of novel metabolites, which may be the potential biomarkers of several metabolic and endocrine disorders. The utilization of this approach could also improve the process of diagnosis and therefore, make treatment more effective. This review article aims to summarize actual and meaningful metabolomic studies in PCOS and point to the potential biomarkers detected in serum, urine, and follicular fluid of the affected women.
\end{abstract}

Keywords: metabolomics; polycystic ovary syndrome (PCOS); metabolites; biomarkers; mass spectrometry

\section{Introduction}

\subsection{Polycystic Ovary Syndrome}

Polycystic ovary syndrome (PCOS) is a complex endocrinopathy, which affects more than $10 \%$ of women of reproductive age [1]. It is the main cause of female infertility due to oligo- or anovulation. Despite such a high incidence, the pathogenesis of PCOS is still unexplained. Some studies suggest that it is due to the genetic factors associated with ovarian steroidogenesis [2]. According to the Androgen Excess and PCOS Society (AE\&PCOS), the diagnosis of PCOS should be based on the presence of clinical and/or biochemical hyperandrogenism (HA) and the ovarian dysfunction defined as menstrual abnormalities (anovulatory oligomenorrhea $(\mathrm{AnO})$ ) or/and the presence of the polycystic ovary morphology (PCOM) in the transvaginal ultrasound (TV-US) [3]. These criteria yield three separate PCOS phenotypes: A, B, and C. Phenotype A includes all the three features (HA, AnO, and PCOM) whereas phenotype B and C only two (HA and AnO or HA and PCOM, respectively). However, regarding Rotterdam criteria, the fourth phenotype (D) was separated to comprise AnO and PCOM presence. The clinical symptoms of hyperandrogenism include hirsutism (present in $60 \%$ of women), androgenic alopecia, and acne, which negatively affect women's psyche, their femininity and lead to low self-esteem and depression [4]. In addition to the reproductive and endocrine dysfunction, PCOS is characterized by intrinsic insulin resistance (IR), which lead to the development of the metabolic syndrome (MetS) and its consequences such as disturbed carbohydrate metabolism 
and type 2 diabetes mellitus (T2DM). Most common clinical manifestation in PCOS is abdominal obesity, which is involved in the development of dyslipidemia, arterial hypertension (AH), as well as non-alcoholic fatty liver disease (NAFLD) [5-8]. These in turn lead to the development of cardiovascular disease (CVD), which still remains the main cause of death among women [9]. The clinical picture of this complex endocrinopathy was presented in Figure 1. Therefore, the treatment of PCOS focuses not only on the symptoms of hyperandrogenism and infertility, but also on improving IR and its metabolic consequences [10]. Thus, there is a need for a better understanding of the pathomechanisms of this complex disorder through the identification of potential biomarkers with the use of new, non-invasive and specific methods. In recent years, one of the developing scientific approaches is metabolomics [11].

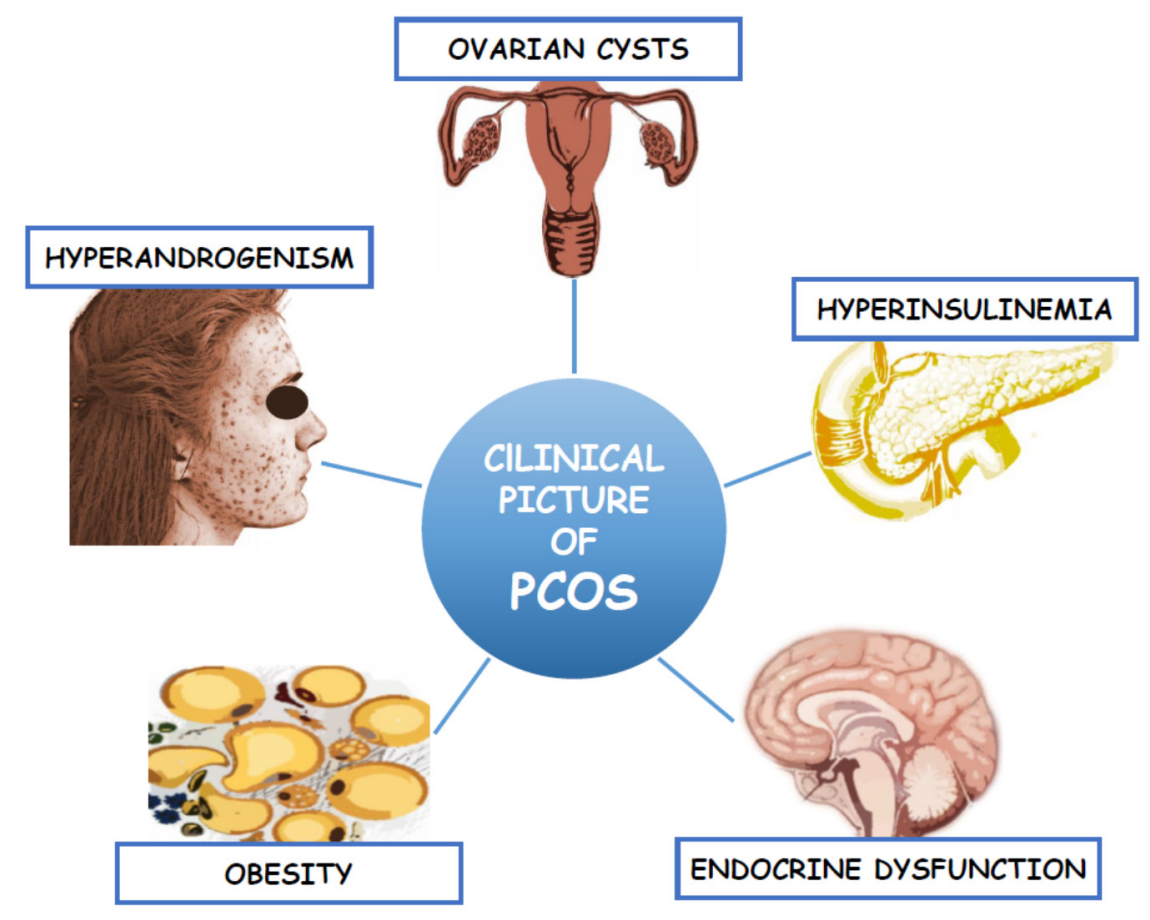

Figure 1. Clinical picture of Polycystic Ovary Syndrome.

\subsection{Metabolomic Approach in Studying the Pathogenesis of Polycystic Ovary Syndrome}

Among "omics" techniques, metabolomics plays an important role in studying the potential mechanisms responsible for the development of PCOS. Metabolomics allows to identify and quantify small molecules, which occur in all living organisms [12]. The set of all human metabolites that have been identified so far is stored in the Human Metabolome Database (HMDB). Each year, the number of identified metabolites grows. Few years ago, about 41,000 metabolites were found, but now this database contains over 114,190 compounds. Among them, the following groups can be found: amino acids, lipids, peptides, vitamins, organic acids and both endo- and exogenous carbohydrates. Therefore, metabolomics serves as a valuable source of information. The metabolome indicates not only a genetically determined phenotype, but also points to the differences determined by other factors, such as age, diet, or physical activity. The application of metabolomics enables monitoring of the state of an organism and provides information on the compounds formed as a result of many biochemical processes. Any disturbances occurring in a living organism cause changes to the qualitative and quantitative profile of the metabolites. The metabolome describes both the physiological and pathological state of the organism. For this reason, it is known to be an attractive approach, compared to genomics and proteomics, which only suggest the presence of metabolic derangements that occur in the organism [13-16]. Due to this fact, the use of metabolomics in studying the pathophysiology of PCOS allows to monitor even the smallest biochemical changes in this endocrinopathy and therefore, may help in its diagnosis [17]. 
Among the many analytical techniques, chromatography coupled with mass spectrometry (MS) seems to be the "gold technique". While chromatography allows for the separation of metabolites present in complex, biological samples, mass spectrometry provides specific information about the chemical structure of the compounds, such as characteristic fragmentation ions, accurate mass, and isotope distribution pattern utilized for the identification of metabolites. MS characterizes very high selectivity and sensitivity that allows to detect and measure trace amounts of metabolites [18]. The combination of MS with gas chromatography (GC-MS) and liquid chromatography (LC-MS) enables to analyse complex biological samples broadly used in metabolomics. GC-MS is suitable for volatile and non-volatile compounds, which require a derivatization step, but first of all thermally stable analytes. The LC-MS technique is widely used for targeted and non-targeted metabolomic analysis and allows to qualify and identify more polar compounds [19]. Nuclear magnetic resonance (NMR), despite its lower sensitivity than MS, allows to analyse metabolites that are difficult to ionize or require derivative reaction for MS and identify compounds with the same masses [20]. A combination of these complementary techniques enables to analyse a broader array of metabolites and offers more certain results than their separate use.

\subsection{Matrices for Metabolomic Studies}

The application of metabolomics allows the use of several matrices such as tissue and body fluids (i.e., plasma, serum, saliva, follicular fluid, semen). The choice of the matrices is associated with the aim of the conducted study as well as the characteristics of the studied disorder. Ovarian tissue can also be used; however, sampling is invasive and problematic. It is usually obtained during laparoscopic wedge resection surgery. For this reason, the use of ovarian tissue in studying the pathophysiology of PCOS is not very common. The matrices widely used in metabolomic studies associated with PCOS are plasma and urine. Serum and urine samples are more common, because they are easily collectible and simple to prepare. On comparing the significantly altered metabolites, it can be observed that the results obtained for both matrices do not completely overlap. The new alternative matrix is follicular fluid, which is innovative in case of PCOS research, especially in terms of oocytes maturation and their quality [21-23].

\section{Metabolic Alterations in PCOS}

PCOS includes a number of abnormalities, which influence several metabolic pathways. It is especially characterized by disturbed metabolism of the steroid hormones, amino acids, carbohydrates, lipids, purines, and the citric acid cycle. Searching for these pathological changes is possible through the metabolomic analyses of biological samples such as serum or plasma, urine, and follicular fluid. Most studies focus on serum and plasma analysis; however, other biological samples also provide substantial information on the existing biochemical derangements. In this review paper, we concentrated on metabolomic studies that were performed in the period of 2014 to 2020 and analysed different biological samples. The PubMed database was searched using the terms "polycystic ovary syndrome" or "PCOS" and "metabolomics". The most important and actual studies using plasma, serum, urine, or FF samples were then analysed.

\subsection{Metabolomic Profile Plasma and Serum Samples}

Murri et al. (2014) published a valuable review where they compared few studies based on the analyses of plasma obtained from PCOS women and healthy controls [11,24-27]. In this paper, we quoted this publication and also presented the results of new studies in this field published since 2014 [28-37]. A set of metabolites found as the most characteristic for PCOS is presented in Table 1. Additionally, information about the applied technique as well as the trend of regulation is included. As can be observed, three metabolic pathways seem to characterise PCOS. Among them, metabolites connected with lipid, amino acid, as well as energy metabolism such as citric acid cycle seem to be the most common. In the case of PCOS, down-regulation of glycerophospholipid metabolism 
and up-regulation of glucose metabolism was observed. The results published by Zhao et al. (2012) show that all of the determined fatty acids are up-regulated in PCOS compared to the control subjects [25]. A contrary phenomenon occurs in the case of phosphatidylcholine (PC), phosphatidylethanolamine (PE) and its derivatives lysophosphatidylcholine (LPC), lysophosphatidylethanolamine (LPE). Metabolites including PC, PE, LPE, and LPC are decreased. In turn, Fan et al. (2019) observed a decreased level of compounds involved in the metabolism of lecithin [36]. In several studies, androgen metabolism was also taken into account. Three major metabolites connected with elevated androgen metabolism were found, namely dehydroepiandrosterone sulphate (DHEAS), dihydrotestosterone sulphate (DHTS), and androsterone sulphate (ANDS) (Table 1). Amino acids (AAs) are the next group of endogenous compounds determined in samples collected from women with PCOS. According to the presented database, there is no homogenous pattern in AAs' regulation. For instance, the levels of arginine, choline, citrulline, glutamate, glycine, and histidine were found to be decreased. In turn, Zhao et al. (2012) reported increased levels of endogenous AAs and glucogenic AAs [25].

Table 1. The most significant changes in metabolites measured in plasma and serum samples in women with PCOS in comparison with control subjects.

\begin{tabular}{|c|c|c|c|c|}
\hline Metabolites & PCOS vs. Control & Metabolic Pathways & Studies & Techniques \\
\hline Cholesterol & $\begin{array}{l}\downarrow \\
\downarrow \\
\downarrow\end{array}$ & Lipid metabolism & $\begin{array}{c}\text { Zhao et al., } 2012 \\
\text { Escobar-Morreale et al., } 2012 \\
\text { Buszewska-Forajta et al., } 2019\end{array}$ & $\begin{array}{l}\text { GC-MS } \\
\text { GC-MS }\end{array}$ \\
\hline Alpha-Tocopherol & $\downarrow$ & Lipid metabolism & Escobar-Morreale et al., 2012 & GC-MS \\
\hline HDL & $\downarrow$ & Lipid metabolism & Zhao et al., 2012 & NMR \\
\hline Phosphatidylcholine & $\begin{array}{l}\downarrow \\
\downarrow \\
\end{array}$ & Lipid metabolism & $\begin{array}{l}\text { Zhao et al., } 2012 \\
\text { Sun et al., } 2012 \\
\end{array}$ & $\begin{array}{l}\text { NMR } \\
\text { NMR }\end{array}$ \\
\hline Linoleic acid & $\begin{array}{l}\uparrow \\
\uparrow \\
\end{array}$ & Lipid metabolism & $\begin{array}{l}\text { Zhao et al., } 2012 \\
\text { Dong et al., } 2015 \\
\end{array}$ & $\begin{array}{l}\text { GC-MS } \\
\text { LC-MS }\end{array}$ \\
\hline Lipoprotein & $\uparrow$ & Lipid metabolism & Zhao et al., 2012 & NMR \\
\hline Palmitic acid & $\uparrow$ & Lipid metabolism & Zhao et al., 2012 & GC-MS \\
\hline C18:0 stearic acid & $\uparrow$ & Lipid metabolism & $\begin{array}{l}\text { Zhao et al., } 2012 \\
\text { Szczuko et al., } 2017\end{array}$ & $\begin{array}{l}\text { GC-MS } \\
\text { GC-MS }\end{array}$ \\
\hline Unsaturated fatty acid & $\uparrow$ & Lipid metabolism & Zhao et al., 2012 & NMR \\
\hline VLDL/LDL & $\uparrow$ & Lipid metabolism & Zhao et al., 2012 & NMR \\
\hline Lipid-CH2CH2CO & $\begin{array}{l}\downarrow \\
\uparrow \\
\end{array}$ & Lipid metabolism & $\begin{array}{c}\text { Atiomo et al., } 2012 \\
\text { Zhao et al., } 2012\end{array}$ & $\begin{array}{l}\text { NMR } \\
\text { NMR }\end{array}$ \\
\hline FFA $16: 1$ & $\uparrow$ & Lipid metabolism & Zhao et al., 2014 & LC-MS \\
\hline FFA 16:2 & $\uparrow$ & Lipid metabolism & Zhao et al., 2014 & LC-MS \\
\hline FFA 18:1 & $\uparrow$ & Lipid metabolism & Zhao et al., 2014 & LC-MS \\
\hline FFA 18:3 & $\uparrow$ & Lipid metabolism & Zhao et al., 2014 & LC-MS \\
\hline FFA 20:1 & $\uparrow$ & Lipid metabolism & Zhao et al., 2014 & LC-MS \\
\hline FFA 20:2 & $\uparrow$ & Lipid metabolism & Zhao et al., 2014 & LC-MS \\
\hline FFA 20:3 & $\uparrow$ & Lipid metabolism & Zhao et al., 2014 & LC-MS \\
\hline FFA 20:4 & $\uparrow$ & Lipid metabolism & Zhao et al., 2014 & LC-MS \\
\hline FFA 20:5 & $\uparrow$ & Lipid metabolism & Zhao et al., 2014 & LC-MS \\
\hline FFA 20:6 & $\uparrow$ & Lipid metabolism & Zhao et al., 2014 & LC-MS \\
\hline FFA 22:5 & $\uparrow$ & Lipid metabolism & Zhao et al., 2014 & LC-MS \\
\hline FFA 22:6 & $\uparrow$ & Lipid metabolism & Zhao et al., 2014 & LC-MS \\
\hline FFA $24: 2$ & $\uparrow$ & Lipid metabolism & Zhao et al., 2014 & LC-MS \\
\hline
\end{tabular}


Table 1. Cont.

\begin{tabular}{|c|c|c|c|c|}
\hline Metabolites & PCOS vs. Control & Metabolic Pathways & Studies & Techniques \\
\hline MG 18:1 & $\uparrow \uparrow$ & Lipid metabolism & Zhao et al., 2014 & LC-MS \\
\hline MG 20:3 & $\uparrow$ & Lipid metabolism & Zhao et al., 2014 & LC-MS \\
\hline $\operatorname{LPC}(16: 1)$ & $\downarrow$ & Lipid metabolism & Zhao et al., 2014 & LC-MS \\
\hline LPC (16:0) & $\downarrow$ & Lipid metabolism & Haoula et al., 2015 & LC-MS \\
\hline LPC (18:0) & $\downarrow$ & Lipid metabolism & Haoula et al., 2015 & LC-MS \\
\hline LPC (18:1) & $\begin{array}{l}\downarrow \\
\downarrow\end{array}$ & Lipid metabolism & $\begin{array}{l}\text { Zhao et al., } 2014 \\
\text { Haoula et al., } 2015\end{array}$ & $\begin{array}{l}\text { LC-MS } \\
\text { LC-MS }\end{array}$ \\
\hline LPC (18:2) & $\begin{array}{l}\downarrow \\
\downarrow \\
\downarrow \\
\uparrow \\
\downarrow\end{array}$ & Lipid metabolism & $\begin{array}{c}\text { Zhao et al., } 2014 \\
\text { Dong et al., } 2015 \\
\text { Jia et al., } 2019 \\
\text { Buszewska-Forajta et al., } 2019 \\
\text { Haoula et al., } 2015\end{array}$ & $\begin{array}{l}\text { LC-MS } \\
\text { LC-MS } \\
\text { LC-MS } \\
\text { LC-MS } \\
\text { LC-MS }\end{array}$ \\
\hline LPC 18:3 & $\begin{array}{l}\downarrow \\
\downarrow\end{array}$ & Lipid metabolism & $\begin{array}{l}\text { Zhao et al., } 2014 \\
\text { Dong et al., } 2015\end{array}$ & $\begin{array}{l}\text { LC-MS } \\
\text { LC-MS }\end{array}$ \\
\hline LPC 20:5 & $\downarrow$ & Lipid metabolism & Zhao et al., 2014 & LC-MS \\
\hline LPC 22:5 & $\downarrow$ & Lipid metabolism & Zhao et al., 2014 & LC-MS \\
\hline LPE 16:0 & $\downarrow$ & Lipid metabolism & Zhao et al., 2014 & LC-MS \\
\hline LPE $18: 1$ & $\downarrow$ & Lipid metabolism & Zhao et al., 2014 & LC-MS \\
\hline LPE 18:2 & $\downarrow$ & Lipid metabolism & Zhao et al., 2014 & LC-MS \\
\hline LPE 20:4 & $\downarrow$ & Lipid metabolism & Zhao et al., 2014 & LC-MS \\
\hline LPE 22:5 & $\begin{array}{l}\downarrow \\
\downarrow \\
\downarrow\end{array}$ & Lipid metabolism & $\begin{array}{c}\text { Zhao et al., } 2014 \\
\text { Dong et al., } 2015 \\
\text { Jia et al., } 2019\end{array}$ & $\begin{array}{l}\text { LC-MS } \\
\text { LC-MS } \\
\text { LC-MS }\end{array}$ \\
\hline PC (18:1/18:4) & $\downarrow$ & Lipid metabolism & Vonica et al., 2019 & LC-MS \\
\hline PC (18:3/18:2) & $\downarrow$ & Lipid metabolism & Vonica et al., 2019 & LC-MS \\
\hline PC (32:4) & $\downarrow$ & Lipid metabolism & Haoula et al., 2015 & LC-MS \\
\hline PC (30:0) & $\downarrow$ & Lipid metabolism & Haoula et al., 2015 & LC-MS \\
\hline PE (42:1) & $\downarrow$ & Lipid metabolism & Haoula et al., 2015 & LC-MS \\
\hline PE (34:0) & $\downarrow$ & Lipid metabolism & Haoula et al., 2015 & LC-MS \\
\hline SM (d18:0/20:2) & $\uparrow$ & Lipid metabolism & Haoula et al., 2015 & LC-MS \\
\hline SM (d18:0/18:0) & $\uparrow$ & Lipid metabolism & Haoula et al., 2015 & LC-MS \\
\hline Triglycerides & $\uparrow$ & Lipid metabolism & Haoula et al., 2015 & LC-MS \\
\hline DG (36:2) & $\uparrow$ & Lipid metabolism & Haoula et al., 2015 & LC-MS \\
\hline DG (36:3) & $\uparrow$ & Lipid metabolism & Haoula et al., 2015 & LC-MS \\
\hline Plasmalogen (30:0) & $\downarrow$ & Lipid metabolism & Haoula et al., 2015 & LC-MS \\
\hline Plasmalogen (40:7) & $\uparrow$ & Lipid metabolism & Haoula et al., 2015 & LC-MS \\
\hline Azelaic acid & $\uparrow$ & Lipid metabolism & Dong et al., 2015 & LC-MS \\
\hline N-undecanoylglycine & $\uparrow$ & Lipid metabolism & Dong et al., 2015 & LC-MS \\
\hline Chenodeoxycholic acid & $\uparrow$ & Lipid metabolism & Fan et al., 2019 & LC-MS \\
\hline Cholic acid & $\downarrow$ & Lipid metabolism & Fan et al., 2019 & LC-MS \\
\hline Clupanodonylcarnitine & $\uparrow$ & Lipid metabolism & Fan et al., 2019 & LC-MS \\
\hline 2-Hydroxylauroylcarnitine & $\uparrow$ & Lipid metabolism & Vonica et al., 2019 & LC-MS \\
\hline Trans-2-dodecenoylcarnitine & $\uparrow$ & Lipid metabolism & Vonica et al., 2019 & LC-MS \\
\hline Cholestane- $3 \beta$ & $\uparrow$ & Sterol lipid metabolism & Vonica et al., 2019 & LC-MS \\
\hline Cholestane- $5 \alpha(18: 0 / 0: 0)$ & $\uparrow$ & Sterol lipid metabolism & Vonica et al., 2019 & LC-MS \\
\hline
\end{tabular}


Table 1. Cont.

\begin{tabular}{|c|c|c|c|c|}
\hline Metabolites & PCOS vs. Control & Metabolic Pathways & Studies & Techniques \\
\hline Cholestane- $6 \beta$-triol & $\uparrow$ & Sterol lipid metabolism & Vonica et al., 2019 & LC-MS \\
\hline Cholestane (18:1/0:0) & $\uparrow$ & Sterol lipid metabolism & Vonica et al., 2019 & LC-MS \\
\hline Androsterone sulphate & $\uparrow$ & $\begin{array}{l}\text { Lipid transport } \\
\text { and metabolism }\end{array}$ & Fan et al., 2019 & LC-MS \\
\hline $11^{\prime}$-Carboxy- $\alpha$-chromanol & $\uparrow$ & $\begin{array}{l}\text { Lipid transport } \\
\text { and metabolism }\end{array}$ & Fan et al., 2019 & LC-MS \\
\hline $\begin{array}{c}\text { (9-cis, } 9^{\prime} \text {-cis)-7, } 77^{\prime}, 8,8^{\prime}- \\
\text { Tetrahydro-y,y-Carotene }\end{array}$ & $\uparrow$ & $\begin{array}{l}\text { Lipid transport } \\
\text { and metabolism }\end{array}$ & Fan et al., 2019 & LC-MS \\
\hline Sphinganine & $\begin{array}{l}\downarrow \\
\downarrow \\
\uparrow\end{array}$ & $\begin{array}{l}\text { Sphingolipid } \\
\text { metabolism }\end{array}$ & $\begin{array}{c}\text { Dong et al., } 2015 \\
\text { Jia et al., } 2019 \\
\text { Buszewska-Forajta et al., } 2019\end{array}$ & $\begin{array}{l}\text { LC-MS } \\
\text { LC-MS } \\
\text { LC-MS }\end{array}$ \\
\hline Phytosphingosine & $\downarrow$ & $\begin{array}{l}\text { Sphingolipid } \\
\text { metabolism }\end{array}$ & Dong et al., 2015 & LC-MS \\
\hline Palmitoylsphingomyelin & $\uparrow$ & $\begin{array}{l}\text { Sphingomyelin } \\
\text { metabolism }\end{array}$ & Fan et al., 2019 & LC-MS \\
\hline SM (d18:1/16:0) & $\uparrow$ & $\begin{array}{l}\text { Sphingomyelin } \\
\text { metabolism }\end{array}$ & Fan et al., 2019 & LC-MS \\
\hline LysoPC (O-18:0) & $\downarrow$ & Lecithin metabolism & Fan et al., 2019 & LC-MS \\
\hline LysoPC (16:0) & $\downarrow$ & Lecithin metabolism & Fan et al., 2019 & LC-MS \\
\hline LysoPC [20:2(11Z,14Z)] & $\downarrow$ & Lecithin metabolism & Fan et al., 2019 & LC-MS \\
\hline Glyceric acid & $\uparrow$ & $\begin{array}{l}\text { Glycerolipid } \\
\text { metabolism }\end{array}$ & Dong et al., 2015 & LC-MS \\
\hline LPC (20:2) & $\downarrow$ & $\begin{array}{l}\text { Glycerophospholipid } \\
\text { metabolism }\end{array}$ & Dong et al., 2015 & LC-MS \\
\hline $\begin{array}{l}\text { 2-Arachidonoyl } \\
\text { glycerophosphocholine }\end{array}$ & $\uparrow$ & $\begin{array}{l}\text { Glycerophospholipid } \\
\text { metabolism }\end{array}$ & Fan et al., 2019 & LC-MS \\
\hline PG [18:1(9Z)/16:0] & $\downarrow$ & $\begin{array}{l}\text { Glycerophospholipid } \\
\text { metabolism }\end{array}$ & Fan et al., 2019 & LC-MS \\
\hline $\begin{array}{c}\text { PE [O-18:1(1Z)/20:4 } \\
(5 Z, 8 Z, 11 Z, 14 Z)]\end{array}$ & $\downarrow$ & $\begin{array}{l}\text { Glycerophospholipid } \\
\text { metabolism }\end{array}$ & Fan et al., 2019 & LC-MS \\
\hline LysoPE [0:0/22:1(13Z)] & $\downarrow$ & $\begin{array}{l}\text { Glycerophospholipid } \\
\text { metabolism }\end{array}$ & Fan et al., 2019 & LC-MS \\
\hline $\begin{array}{c}\text { PE [O-16:1(1Z)/22:6 } \\
(4 Z, 7 Z, 10 Z, 13 Z, 16 Z, 19 Z)] \\
\end{array}$ & $\downarrow$ & $\begin{array}{l}\text { Glycerophospholipid } \\
\text { metabolism }\end{array}$ & Fan et al., 2019 & LC-MS \\
\hline $\begin{array}{c}\mathrm{PE} \\
{[22: 4(7 \mathrm{Z}, 10 \mathrm{Z}, 13 \mathrm{Z}, 16 \mathrm{Z}) / 16: 0]}\end{array}$ & $\downarrow$ & $\begin{array}{l}\text { Glycerophospholipid } \\
\text { metabolism }\end{array}$ & Fan et al., 2019 & LC-MS \\
\hline $\begin{array}{c}\mathrm{PC} \\
{[16: 1(9 \mathrm{Z}) / 22: 2(13 \mathrm{Z}, 16 \mathrm{Z})]}\end{array}$ & $\downarrow$ & $\begin{array}{l}\text { Glycerophospholipid } \\
\text { metabolism }\end{array}$ & Fan et al., 2019 & LC-MS \\
\hline PG (18:0/16:0) & $\uparrow$ & $\begin{array}{l}\text { Glycerophospholipid } \\
\text { metabolism }\end{array}$ & Fan et al., 2019 & LC-MS \\
\hline PG (18:1(9Z)/18:0) & $\downarrow$ & $\begin{array}{l}\text { Glycerophospholipid } \\
\text { metabolism }\end{array}$ & Fan et al., 2019 & LC-MS \\
\hline DG (18:1n9/0:0/20:4n3) & $\uparrow$ & $\begin{array}{l}\text { Diacyloglycerol } \\
\text { metabolism }\end{array}$ & Fan et al., 2019 & LC-MS \\
\hline TG (18:2/18:2/0-18:0) & $\uparrow$ & $\begin{array}{l}\text { Diacyloglycerol } \\
\text { metabolism }\end{array}$ & Vonica et al., 2019 & LC-MS \\
\hline DG (22:2/0:0/22:4) & $\downarrow$ & $\begin{array}{l}\text { Diacyloglycerol } \\
\text { metabolism }\end{array}$ & Vonica et al., 2019 & LC-MS \\
\hline Arginine & $\downarrow$ & $\begin{array}{l}\text { Amino acids } \\
\text { metabolism }\end{array}$ & $\begin{array}{l}\text { Atiomo et al., } 2012 \\
\text { Sun et al., } 2012\end{array}$ & $\begin{array}{l}\text { NMR } \\
\text { NMR }\end{array}$ \\
\hline
\end{tabular}


Table 1. Cont.

\begin{tabular}{|c|c|c|c|c|}
\hline Metabolites & PCOS vs. Control & Metabolic Pathways & Studies & Techniques \\
\hline Choline & $\downarrow$ & $\begin{array}{l}\text { Amino acids } \\
\text { metabolism }\end{array}$ & Sun et al., 2012 & NMR \\
\hline Citruline & $\downarrow$ & $\begin{array}{l}\text { Amino acids } \\
\text { metabolism }\end{array}$ & Atiomo et al., 2012 & NMR \\
\hline Glutamate & $\downarrow$ & $\begin{array}{l}\text { Amino acids } \\
\text { metabolism }\end{array}$ & Atiomo et al., 2012 & NMR \\
\hline $\begin{array}{l}\text { Glycerophosphocholine/ } \\
\text { phosphocholine }\end{array}$ & $\downarrow$ & $\begin{array}{l}\text { Amino acids } \\
\text { metabolism }\end{array}$ & Sun et al., 2012 & NMR \\
\hline Glycine & $\downarrow$ & $\begin{array}{l}\text { Amino acids } \\
\text { metabolism }\end{array}$ & Zhao et al., 2012 & GC-MS \\
\hline Histidine & $\downarrow$ & $\begin{array}{l}\text { Amino acids } \\
\text { metabolism }\end{array}$ & $\begin{array}{l}\text { Atiomo et al., } 2012 \\
\text { RoyChoudhury et al., } 2016\end{array}$ & NMR \\
\hline AAA & $\uparrow$ & $\begin{array}{l}\text { Amino acids } \\
\text { metabolism }\end{array}$ & Zhao et al., 2012 & GC-MS \\
\hline BCAA & $\uparrow$ & $\begin{array}{l}\text { Amino acids } \\
\text { metabolism }\end{array}$ & Zhao et al., 2012 & GC-MS \\
\hline BCAA/AAA & $\downarrow$ & $\begin{array}{l}\text { Amino acids } \\
\text { metabolism }\end{array}$ & Zhao et al., 2012 & GC-MS \\
\hline Aspartate & $\uparrow$ & $\begin{array}{l}\text { Amino acids } \\
\text { metabolism }\end{array}$ & Zhao et al., 2012 & GC-MS \\
\hline Endogenous AAs & $\uparrow$ & $\begin{array}{l}\text { Amino acids } \\
\text { metabolism }\end{array}$ & Zhao et al., 2012 & GC-MS \\
\hline Gluconeogenic AAs & $\uparrow$ & $\begin{array}{l}\text { Amino acids } \\
\text { metabolism }\end{array}$ & Zhao et al., 2012 & GC-MS \\
\hline Serine & $\uparrow$ & $\begin{array}{l}\text { Amino acids } \\
\text { metabolism }\end{array}$ & Zhao et al., 2012 & GC-MS \\
\hline 2-Aminobutyrate & $\uparrow$ & $\begin{array}{l}\text { Amino acid } \\
\text { metabolism }\end{array}$ & Whigham et al., 2014 & NMR \\
\hline 2-Hydroxybutyrate & $\uparrow$ & $\begin{array}{l}\text { Amino acid } \\
\text { metabolism }\end{array}$ & Whigham et al., 2014 & NMR \\
\hline 2-Hyroxyisovalerate & $\uparrow$ & $\begin{array}{l}\text { Amino acid } \\
\text { metabolism }\end{array}$ & Whigham et al., 2014 & NMR \\
\hline 2-Oxocaproate & $\uparrow$ & $\begin{array}{l}\text { Amino acid } \\
\text { metabolism }\end{array}$ & Whigham et al., 2014 & NMR \\
\hline 2-Oxoisocaproate & $\uparrow$ & $\begin{array}{l}\text { Amino acid } \\
\text { metabolism }\end{array}$ & Whigham et al., 2014 & NMR \\
\hline 3-Hydroxybutyrate & $\uparrow$ & $\begin{array}{l}\text { Amino acid } \\
\text { metabolism }\end{array}$ & Whigham et al., 2014 & NMR \\
\hline 3-Methyl-2-oxovalerate & $\uparrow$ & $\begin{array}{l}\text { Amino acid } \\
\text { metabolism }\end{array}$ & Whigham et al., 2014 & NMR \\
\hline Betadine & $\uparrow$ & $\begin{array}{l}\text { Amino acid } \\
\text { metabolism }\end{array}$ & Whigham et al., 2014 & NMR \\
\hline Creatinine & $\uparrow$ & $\begin{array}{l}\text { Amino acid } \\
\text { metabolism }\end{array}$ & $\begin{array}{l}\text { Whigham et al., } 2014 \\
\text { Sun et al., } 2012\end{array}$ & $\begin{array}{l}\text { NMR } \\
\text { NMR }\end{array}$ \\
\hline Dimethylamine & $\uparrow$ & $\begin{array}{l}\text { Amino acid } \\
\text { metabolism }\end{array}$ & Whigham et al., 2014 & NMR \\
\hline Lysine & $\begin{array}{l}\uparrow \\
\uparrow \\
\downarrow\end{array}$ & $\begin{array}{l}\text { Amino acid } \\
\text { metabolism }\end{array}$ & $\begin{array}{c}\text { Whigham et al., } 2014 \\
\text { Zhao et al., } 2012 \\
\text { Atiomo et al., } 2012\end{array}$ & $\begin{array}{c}\text { NMR } \\
\text { GC-MS } \\
\text { NMR } \\
\end{array}$ \\
\hline Methionine & $\begin{array}{l}\uparrow \\
\downarrow\end{array}$ & $\begin{array}{l}\text { Amino acid } \\
\text { metabolism }\end{array}$ & $\begin{array}{l}\text { Whigham et al., } 2014 \\
\text { Sun et al., } 2012\end{array}$ & $\begin{array}{l}\text { NMR } \\
\text { NMR }\end{array}$ \\
\hline
\end{tabular}


Table 1. Cont.

\begin{tabular}{|c|c|c|c|c|}
\hline Metabolites & PCOS vs. Control & Metabolic Pathways & Studies & Techniques \\
\hline Ornithine & $\begin{array}{l}\uparrow \\
\uparrow \\
\downarrow\end{array}$ & $\begin{array}{l}\text { Amino acid } \\
\text { metabolism }\end{array}$ & $\begin{array}{l}\text { Whigham et al., } 2014 \\
\text { Zhao et al., } 2012 \\
\text { Atiomo et al., } 2012 \\
\end{array}$ & $\begin{array}{c}\text { NMR } \\
\text { GC-MS } \\
\text { NMR } \\
\end{array}$ \\
\hline Sarcosine & $\uparrow$ & $\begin{array}{l}\text { Amino acid } \\
\text { metabolism }\end{array}$ & Whigham et al., 2014 & NMR \\
\hline Taurine & $\uparrow$ & $\begin{array}{l}\text { Amino acid } \\
\text { metabolism }\end{array}$ & Whigham et al., 2014 & NMR \\
\hline Tryptophan & $\begin{array}{l}\uparrow \\
\uparrow \\
\uparrow \\
\end{array}$ & $\begin{array}{l}\text { Amino acid } \\
\text { metabolism }\end{array}$ & $\begin{array}{c}\text { Whigham et al., } 2014 \\
\quad \text { Zhao et al., } 2012 \\
\text { Buszewska-Forajta et al., } 2019\end{array}$ & $\begin{array}{c}\text { NMR } \\
\text { GC-MS } \\
\text { GC/LC-MS }\end{array}$ \\
\hline Tyrosine & $\begin{array}{l}\uparrow \\
\uparrow \\
\uparrow \\
\end{array}$ & $\begin{array}{l}\text { Amino acid } \\
\text { metabolism }\end{array}$ & $\begin{array}{c}\text { Whigham et al., } 2014 \\
\text { Zhao et al., } 2012 \\
\text { Buszewska-Forajta et al., } 2019\end{array}$ & $\begin{array}{l}\text { NMR } \\
\text { GC-MS } \\
\text { GC-MS }\end{array}$ \\
\hline Glutamate & $\begin{array}{l}\downarrow \\
\uparrow\end{array}$ & $\begin{array}{l}\text { Amino acids } \\
\text { metabolism }\end{array}$ & $\begin{array}{l}\text { RoyChoudhury et al., } 2016 \\
\text { Whigham et al., } 2014\end{array}$ & $\begin{array}{l}\text { NMR } \\
\text { NMR }\end{array}$ \\
\hline Glutamine & $\begin{array}{l}\downarrow \\
\uparrow \\
\downarrow\end{array}$ & $\begin{array}{l}\text { Amino acids } \\
\text { metabolism }\end{array}$ & $\begin{array}{l}\text { RoyChoudhury et al., } 2016 \\
\text { Whigham et al., } 2014 \\
\text { Sun et al., } 2012\end{array}$ & $\begin{array}{l}\text { NMR } \\
\text { NMR } \\
\text { NMR }\end{array}$ \\
\hline Proline & $\begin{array}{l}\downarrow \\
\downarrow \\
\downarrow \\
\uparrow\end{array}$ & $\begin{array}{l}\text { Amino acids } \\
\text { metabolism }\end{array}$ & $\begin{array}{c}\text { Atiomo et al., } 2012 \\
\text { Zhao et al., } 2012 \\
\text { RoyChoudhury et al., } 2016 \\
\text { Whigham et al., } 2014\end{array}$ & $\begin{array}{l}\text { NMR } \\
\text { GC-MS } \\
\text { NMR } \\
\text { NMR }\end{array}$ \\
\hline Alanine & $\begin{array}{l}\uparrow \\
\uparrow \\
\uparrow \\
\downarrow\end{array}$ & $\begin{array}{l}\text { Amino acids } \\
\text { metabolism }\end{array}$ & $\begin{array}{c}\text { RoyChoudhury et al., } 2016 \\
\text { Whigham et al., } 2014 \\
\text { Zhao et al., } 2012 \\
\text { Escobar-Morreale et al., } 2012\end{array}$ & $\begin{array}{l}\text { NMR } \\
\text { NMR } \\
\text { NMR } \\
\text { GC-MS }\end{array}$ \\
\hline Leucine & $\begin{array}{l}\uparrow \\
\uparrow \\
\downarrow \\
\uparrow\end{array}$ & $\begin{array}{l}\text { Amino acids } \\
\text { metabolism }\end{array}$ & $\begin{array}{c}\text { RoyChoudhury et al., } 2016 \\
\text { Whigham et al., } 2014 \\
\text { Sun et al., } 2012 \\
\text { Zhao et al., } 2012 \\
\end{array}$ & $\begin{array}{l}\text { NMR } \\
\text { NMR } \\
\text { NMR } \\
\text { GC-MS }\end{array}$ \\
\hline Isoleucine & $\begin{array}{l}\uparrow \\
\downarrow\end{array}$ & $\begin{array}{l}\text { Amino acids } \\
\text { metabolism }\end{array}$ & $\begin{array}{l}\text { Whigham et al., } 2014 \\
\text { Zhao et al., } 2012\end{array}$ & $\begin{array}{l}\text { NMR } \\
\text { GC-MS }\end{array}$ \\
\hline Valine & $\begin{array}{l}\uparrow \\
\uparrow \\
\uparrow\end{array}$ & $\begin{array}{l}\text { Amino acids } \\
\text { metabolism }\end{array}$ & $\begin{array}{l}\text { RoyChoudhury et al., } 2016 \\
\text { Whigham et al., } 2014 \\
\text { Zhao et al., } 2012\end{array}$ & $\begin{array}{l}\text { NMR } \\
\text { NMR } \\
\text { GC-MS }\end{array}$ \\
\hline Threonine & $\begin{array}{l}\uparrow \\
\uparrow \\
\uparrow \\
\uparrow\end{array}$ & $\begin{array}{l}\text { Amino acids } \\
\text { metabolism }\end{array}$ & $\begin{array}{c}\text { RoyChoudhury et al., } 2016 \\
\text { Whigham et al., } 2014 \\
\text { Zhao et al., } 2012 \\
\text { Buszewska-Forajta et al., } 2019\end{array}$ & $\begin{array}{c}\text { NMR } \\
\text { NMR } \\
\text { GC-MS } \\
\text { GC-MS }\end{array}$ \\
\hline Cysteine-S-sulphate & $\uparrow$ & $\begin{array}{l}\text { Amino acid } \\
\text { metabolism }\end{array}$ & Fan et al., 2019 & LC-MS \\
\hline Glu-Glu & $\uparrow$ & $\begin{array}{l}\text { Amino acid } \\
\text { metabolism }\end{array}$ & Dong et al., 2015 & LC-MS \\
\hline Asparagine & $\uparrow$ & $\begin{array}{l}\text { Amino acid } \\
\text { metabolism }\end{array}$ & Whigham et al., 2014 & NMR \\
\hline Ketoleucine & $\downarrow$ & $\begin{array}{l}\text { Valine, leucine, } \\
\text { and isoleucine } \\
\text { degradation }\end{array}$ & Dong et al., 2015 & LC-MS \\
\hline Glutamic acidc & $\uparrow$ & $\begin{array}{c}\text { Glutamate metabolism, } \\
\text { amino sugar } \\
\text { metabolism }\end{array}$ & Dong et al., 2015 & LC-MS \\
\hline Phenylpyruvic acid & $\uparrow$ & $\begin{array}{l}\text { Phenylalanine } \\
\text { and tyrosine } \\
\text { metabolism }\end{array}$ & Dong et al., 2015 & LC-MS \\
\hline Gly.Phe & $\uparrow$ & $\begin{array}{l}\text { Phenylalanine } \\
\text { and tyrosine } \\
\text { metabolism }\end{array}$ & Zhao et al., 2014 & LC-MS \\
\hline
\end{tabular}


Table 1. Cont.

\begin{tabular}{|c|c|c|c|c|}
\hline Metabolites & PCOS vs. Control & Metabolic Pathways & Studies & Techniques \\
\hline Phenylalanine & $\begin{array}{l}\uparrow \\
\uparrow \\
\uparrow \\
\uparrow\end{array}$ & $\begin{array}{l}\text { Phenylalanine } \\
\text { and tyrosine } \\
\text { metabolism }\end{array}$ & $\begin{array}{c}\text { Zhao et al., } 2014 \\
\text { Whigham et al., } 2014 \\
\text { Zhao et al., } 2012 \\
\text { Buszewska-Forajta et al., } 2019\end{array}$ & $\begin{array}{l}\text { LC-MS } \\
\text { NMR } \\
\text { GC-MS } \\
\text { GC-MS }\end{array}$ \\
\hline Phe-Phe & $\uparrow$ & $\begin{array}{l}\text { Phenylalanine } \\
\text { and tyrosine } \\
\text { metabolism }\end{array}$ & Zhao et al., 2014 & LC-MS \\
\hline Kynurenine & $\downarrow$ & $\begin{array}{l}\text { Tryptophan } \\
\text { metabolism }\end{array}$ & Zhao et al., 2014 & LC-MS \\
\hline 5-Hydroxyindoleacetic acid & $\downarrow$ & $\begin{array}{l}\text { Tryptophan } \\
\text { metabolism }\end{array}$ & Dong et al., 2015 & LC-MS \\
\hline Homoserine & $\begin{array}{l}\downarrow \\
\uparrow\end{array}$ & $\begin{array}{l}\text { Methionine } \\
\text { metabolism }\end{array}$ & $\begin{array}{c}\text { Zhao et al., } 2014 \\
\text { Whigham et al., } 2014\end{array}$ & $\begin{array}{l}\text { LC-MS } \\
\text { NMR }\end{array}$ \\
\hline S-Adenosylmethionine & $\downarrow$ & $\begin{array}{l}\text { Thiol amino acid } \\
\text { metabolic cycle }\end{array}$ & Fan et al., 2019 & LC-MS \\
\hline Pyroglutamic acid & $\uparrow$ & $\begin{array}{l}\text { Glutathione } \\
\text { metabolism }\end{array}$ & Dong et al., 2015 & LC-MS \\
\hline Lysyl-albumin & $\downarrow$ & Protein metabolism & Zhao et al., 2012 & NMR \\
\hline Trimethylamine $\mathrm{N}$-oxide & $\downarrow$ & Protein metabolism & Sun et al., 2012 & NMR \\
\hline 2-Ketoisocaproic acid & $\downarrow$ & Protein metabolism & Escobar-Morreale et al., 2012 & GC-MS \\
\hline Dimethylamine & $\uparrow$ & Protein metabolism & Sun et al., 2012 & NMR \\
\hline $\mathrm{N}$-acetylglycoprotein & $\begin{array}{l}\downarrow \\
\uparrow \\
\end{array}$ & Protein metabolism & $\begin{array}{l}\text { Zhao et al., } 2012 \\
\text { Sun et al., } 2012 \\
\end{array}$ & $\begin{array}{l}\text { NMR } \\
\text { NMR }\end{array}$ \\
\hline Hypoxanthine & $\uparrow$ & Purine metabolism & Zhao et al., 2014 & LC-MS \\
\hline Inosine & $\uparrow$ & Purine metabolism & Zhao et al., 2014 & LC-MS \\
\hline Allantoic acid & $\uparrow$ & Purine metabolism & Dong et al., 2015 & LC-MS \\
\hline Uric acid & $\begin{array}{l}\uparrow \\
\uparrow\end{array}$ & Purine metabolism & $\begin{array}{c}\text { Zhao et al., } 2012 \\
\text { Buszewska-Forajta et al., } 2019\end{array}$ & $\begin{array}{c}\text { GC-MS } \\
\text { GC/LC-MS }\end{array}$ \\
\hline Cyclic GMP & $\uparrow$ & Purine metabolism & Fan et al., 2019 & LC-MS \\
\hline Uridine & $\downarrow$ & $\begin{array}{l}\text { Pyrimidine } \\
\text { metabolism }\end{array}$ & $\begin{array}{l}\text { Zhao et al., } 2014 \\
\text { Dong et al., } 2015\end{array}$ & $\begin{array}{l}\text { LC-MS } \\
\text { LC-MS }\end{array}$ \\
\hline 5,6-Dihydrouridine & $\uparrow$ & $\begin{array}{l}\text { Pyrimidine metabolic } \\
\text { cycle }\end{array}$ & Fan et al., 2019 & LC-MS \\
\hline DHEAS & $\begin{array}{l}\uparrow \\
\uparrow \\
\uparrow \\
\uparrow \\
\uparrow \\
\end{array}$ & Androgen metabolism & $\begin{array}{c}\text { Zhao et al., } 2014 \\
\text { Dong et al., } 2015 \\
\text { Buszewska-Forajta et al., } 2019 \\
\text { Jia et al., } 2019 \\
\text { Fan et al., } 2019\end{array}$ & $\begin{array}{l}\text { LC-MS } \\
\text { LC-MS } \\
\text { LC-MS } \\
\text { LC-MS } \\
\text { LC-MS }\end{array}$ \\
\hline ANDS & $\uparrow$ & Androgen metabolism & Zhao et al., 2014 & LC-MS \\
\hline DHTS & $\uparrow$ & Androgen metabolism & Zhao et al., 2014 & LC-MS \\
\hline Pregnenolone sulphate & $\downarrow$ & $\begin{array}{l}\text { Steroid hormone } \\
\text { biosynthesis }\end{array}$ & Dong et al., 2015 & LC-MS \\
\hline 19-Oxotestosterone & $\uparrow$ & $\begin{array}{l}\text { Steroid hormone } \\
\text { biosynthesis }\end{array}$ & Dong et al., 2015 & LC-MS \\
\hline C10:0 lauric acid & $\downarrow$ & Fatty acid metabolism & Szczuko et al., 2017 & GC-MS \\
\hline C15:0 pentadecanoic acid & $\downarrow$ & Fatty acid metabolism & Szczuko et al., 2017 & GC-MS \\
\hline $\begin{array}{c}\text { C15:1 cis-10- } \\
\text { pentadecanoic acid }\end{array}$ & $\downarrow$ & Fatty acid metabolism & Szczuko et al., 2017 & GC-MS \\
\hline C17:0 heptadecanoic acid & $\downarrow$ & Fatty acid metabolism & Szczuko et al., 2017 & GC-MS \\
\hline C20:0 arachidic acid & $\downarrow$ & Fatty acid metabolism & Szczuko et al., 2017 & GC-MS \\
\hline
\end{tabular}


Table 1. Cont.

\begin{tabular}{|c|c|c|c|c|}
\hline Metabolites & PCOS vs. Control & Metabolic Pathways & Studies & Techniques \\
\hline $\begin{array}{l}\text { C20:1 cis- } 11- \\
\text { eicosanoic acid }\end{array}$ & $\uparrow$ & Fatty acid metabolism & Szczuko et al., 2017 & GC-MS \\
\hline C22:5 EPA & $\downarrow$ & Fatty acid metabolism & Szczuko et al., 2017 & GC-MS \\
\hline C22:0 behenic acid & $\downarrow$ & Fatty acid metabolism & Szczuko et al., 2017 & GC-MS \\
\hline C23:0 tricosanoic acid & $\downarrow$ & Fatty acid metabolism & Szczuko et al., 2017 & GC-MS \\
\hline $\begin{array}{c}\text { C22:4n6 } \\
\text { docosatetraenic acid }\end{array}$ & $\uparrow$ & Fatty acid metabolism & Szczuko et al., 2017 & GC-MS \\
\hline C24:0 lignoceric acid & $\downarrow$ & Fatty acid metabolism & Szczuko et al., 2017 & GC-MS \\
\hline C24:1 nervonic acid & $\uparrow$ & Fatty acid metabolism & Szczuko et al., 2017 & GC-MS \\
\hline 9-HODE/13-HODE & $\uparrow$ & Fatty acid metabolism & Dong et al., 2015 & LC-MS \\
\hline$\alpha$-Linolenic acid & $\uparrow$ & Fatty acid metabolism & Dong et al., 2015 & LC-MS \\
\hline C18:2n6c linoleic acid & $\downarrow$ & Fatty acid metabolism & Szczuko et al., 2017 & GC-MS \\
\hline Vaccenic acid & $\uparrow$ & Fatty acid metabolism & Dong et al., 2015 & LC-MS \\
\hline Docosatrienoic acid & $\uparrow$ & Fatty acid metabolism & Dong et al., 2015 & LC-MS \\
\hline Eicosapentaenoic acid & $\uparrow$ & Fatty acid metabolism & Dong et al., 2015 & LC-MS \\
\hline Galbanic acid & $\uparrow$ & Fatty acid metabolism & Fan et al., 2019 & LC-MS \\
\hline C14:0 myristic acid & $\begin{array}{l}\uparrow \\
\downarrow\end{array}$ & Fatty acid biosynthesis & $\begin{array}{l}\text { Dong et al., } 2015 \\
\text { Szczuko et al., } 2017\end{array}$ & $\begin{array}{l}\text { LC-MS } \\
\text { GC-MS }\end{array}$ \\
\hline Palmitoleic acid & $\uparrow$ & Fatty acid biosynthesis & Dong et al., 2015 & LC-MS \\
\hline Palmitoleoylethanolamide & $\uparrow$ & $\begin{array}{l}\text { Fatty acid amide } \\
\text { metabolism }\end{array}$ & Dong et al., 2015 & LC-MS \\
\hline Oleamide & $\begin{array}{l}\uparrow \\
\uparrow \\
\end{array}$ & $\begin{array}{l}\text { Fatty acid amide } \\
\text { metabolism }\end{array}$ & $\begin{array}{l}\text { Zhao et al., } 2014 \\
\text { Dong et al., } 2015\end{array}$ & $\begin{array}{l}\text { LC-MS } \\
\text { LC-MS }\end{array}$ \\
\hline Palmitic amide & $\begin{array}{l}\uparrow \\
\uparrow \\
\end{array}$ & $\begin{array}{l}\text { Fatty acid amide } \\
\text { metabolism }\end{array}$ & $\begin{array}{l}\text { Zhao et al., } 2014 \\
\text { Dong et al., } 2015\end{array}$ & $\begin{array}{l}\text { LC-MS } \\
\text { LC-MS }\end{array}$ \\
\hline PEA & $\uparrow$ & $\begin{array}{l}\text { Fatty acid amide } \\
\text { metabolism }\end{array}$ & Zhao et al., 2014 & LC-MS \\
\hline AEA & $\uparrow$ & $\begin{array}{l}\text { Fatty acid amide } \\
\text { metabolism }\end{array}$ & Zhao et al., 2014 & LC-MS \\
\hline Carnitine C2:0 & $\uparrow$ & $\begin{array}{l}\text { Beta oxidation of fatty } \\
\text { acids }\end{array}$ & Zhao et al., 2014 & LC-MS \\
\hline Carnitine C6:0 & $\uparrow$ & $\begin{array}{l}\text { Beta oxidation of fatty } \\
\text { acids }\end{array}$ & Zhao et al., 2014 & LC-MS \\
\hline Carnitine C18 & $\uparrow$ & $\begin{array}{l}\text { Beta oxidation of fatty } \\
\text { acids }\end{array}$ & Zhao et al., 2014 & LC-MS \\
\hline Carnitine & $\downarrow$ & Oxidation of fatty acids & $\begin{array}{c}\text { Dong et al., } 2015 \\
\text { Jia et al., } 2019\end{array}$ & $\begin{array}{l}\text { LC-MS } \\
\text { LC-MS }\end{array}$ \\
\hline Glycocholic acid & $\downarrow$ & Bile acid metabolism & $\begin{array}{c}\text { Zhao et al., } 2014 \\
\text { Jia et al., } 2019\end{array}$ & $\begin{array}{l}\text { LC-MS } \\
\text { LC-MS }\end{array}$ \\
\hline $\begin{array}{l}\text { 3,7-Dihydroxy-5- } \\
\text { cholestenoic acid }\end{array}$ & $\uparrow$ & Bile acid metabolism & Fan et al., 2019 & LC-MS \\
\hline $\begin{array}{l}\text { 3- } \beta \text {-Hydroxy-4- } \beta \text {-methyl-5- } \\
\alpha \text {-cholest-7-ene-4- } \alpha \text {-carboxylate }\end{array}$ & te & Bile acid metabolism & Fan et al., 2019 & LC-MS \\
\hline Formate & $\uparrow$ & Pyruvate metabolism & Whigham et al., 2014 & NMR \\
\hline Fructose & $\uparrow$ & Pyruvate metabolism & Whigham et al., 2014 & NMR \\
\hline Mannose & $\uparrow$ & Pyruvate metabolism & Whigham et al., 2014 & NMR \\
\hline
\end{tabular}


Table 1. Cont.

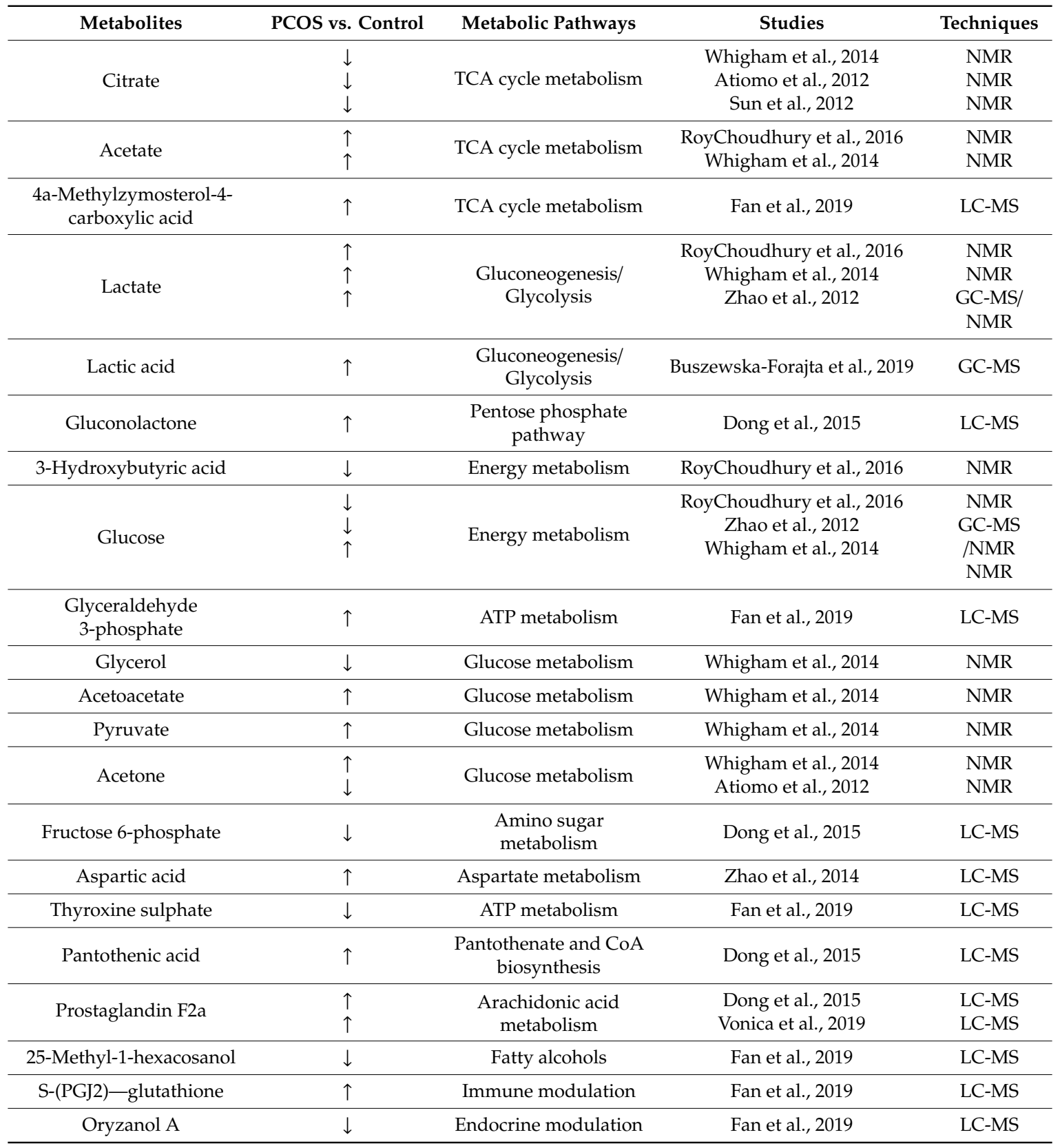

$\mathrm{HDL}=$ high-density lipoproteins, VLDL/LDL = very-low density lipoproteins/ low density lipoproteins, FFA = free fatty acid, PC = phosphatidylcholine, PE = phosphatidylethanolamine, LPC $($ LysoPC $)=$ lysophosphatidylcholine, $\mathrm{LPE}=$ lysophospha-tidylethanolamine, $\mathrm{SM}=$ sphingomyelin, $\mathrm{DG}=$ diglyceride, $\mathrm{TG}=$ trigliceride, PG = phosphatidylglycerol, AAA = aromatic amino acids, BCAA = branched-chain amino acid, AAs = amino acid, GMP = guanosine monophosphate, DHEAS = dehydro-epiandrosterone sulphate, ANDS = androsterone sulphate, DHTS = dihydrotestosterone sulphate, EPA = eicosapentaenoic acid, HODE $=$ hydroxyoctadecadienoic acid, $\mathrm{PEA}=$ palmitoylethanolamide, AEA = N-arachidonoylethanolamine; $\uparrow$ up-regulation; $\downarrow$ down-regulation.

\subsection{Metabolomic Profile of the Urine Samples}

There are relatively few metabolomic studies on PCOS where urine is used as the biological matrix. Urine samples are a convenient study material due to non-invasive sampling as well as easy sample preparation because of the lower content of protein compared to serum and plasma [38]. This matrix is also rich in metabolites of the metabolic pathways, which may be deranged in PCOS. Metabolites, which were down- and up-regulated in women with PCOS compared to healthy controls, are presented in Table 2 [39-41]. Zou et al. (2018) reported that some carbohydrates and fatty acids metabolites are 
up-regulated in PCOS in comparison with the control subjects [40]. A contrary phenomenon occurs in the case of glycerolipids, where levels of 5 out of 7 compounds are decreased, while up-regulation of triglyceride (TG) and DG (16:1(9Z)/14:0/0:0) was reported. Dhayat et al. (2018) focused on the determination of androgens in PCOS, which were all elevated [41]. A similar trend is reported for AAs, glucocorticoids, and peptides.

Table 2. The most significant changes in urinary metabolites in women with PCOS in comparison with the control subjects.

\begin{tabular}{|c|c|c|c|c|}
\hline Metabolites & PCOS vs. Control & Metabolic Pathways & Studies & Techniques \\
\hline Lactose & $\uparrow$ & $\begin{array}{l}\text { Carbohydrate } \\
\text { metabolism }\end{array}$ & Zou et al., 2018 & GC-MS \\
\hline Gluconic acid & $\uparrow$ & $\begin{array}{l}\text { Carbohydrate } \\
\text { metabolism }\end{array}$ & Zou et al., 2018 & GC-MS \\
\hline 3-hydroxypropionic acid & $\uparrow$ & $\begin{array}{l}\text { Carbohydrate } \\
\text { metabolism }\end{array}$ & Zou et al., 2018 & GC-MS \\
\hline Arabinitol & $\uparrow$ & $\begin{array}{l}\text { Carbohydrate } \\
\text { metabolism }\end{array}$ & Zou et al., 2018 & GC-MS \\
\hline Fucose & $\uparrow$ & $\begin{array}{l}\text { Carbohydrate } \\
\text { metabolism }\end{array}$ & Zou et al., 2018 & GC-MS \\
\hline Oxalic acid & $\uparrow$ & $\begin{array}{l}\text { Carbohydrate } \\
\text { metabolism }\end{array}$ & Zou et al., 2018 & GC-MS \\
\hline Arabic candy & $\uparrow$ & Lipid metabolism & Zou et al., 2018 & GC-MS \\
\hline Stearic acid & $\uparrow$ & Lipid metabolism & Zou et al., 2018 & GC-MS \\
\hline Palmitic acid & $\uparrow$ & Lipid metabolism & Zou et al., 2018 & GC-MS \\
\hline Phosphoethanolamine & $\uparrow$ & Lipid metabolism & Zou et al., 2018 & GC-MS \\
\hline 2-(14,15-Epoxyeicosatrienoyl) & $\downarrow$ & Glycerolipids & Wang et al., 2015 & LC-MS \\
\hline $\begin{array}{l}\text { TG (14:1(9Z)/14:0/ } \\
\text { 22:2(13Z,16Z)) }\end{array}$ & $\downarrow$ & Glycerolipids & Wang et al., 2015 & LC-MS \\
\hline TG (14:0/24:1(15Z)/14:1(9Z)) & $\downarrow$ & Glycerolipids & Wang et al., 2015 & LC-MS \\
\hline TG(16:0/14:0/18:0) & $\downarrow$ & Glycerolipids & Wang et al., 2015 & LC-MS \\
\hline TG (16:0/14:1(9Z)/20:1(11Z)) & $\downarrow$ & Glycerolipids & Wang et al., 2015 & LC-MS \\
\hline TG & $\uparrow$ & Glycerolipids & Wang et al., 2015 & LC-MS \\
\hline DG (16:1(9Z)/14:0/0:0) & $\uparrow$ & Glycerolipids & Wang et al., 2015 & LC-MS \\
\hline PC (22:2(13Z,16Z)/18:1(9Z)) & $\downarrow$ & Glycerophospholipids & Wang et al., 2015 & LC-MS \\
\hline PC (14:1(9Z)/14:1(9Z)) & $\downarrow$ & Glycerophospholipids & Wang et al., 2015 & LC-MS \\
\hline LPA (16:0/0:0) & $\downarrow$ & Glycerophospholipids & Wang et al., 2015 & LC-MS \\
\hline PE (14:1(9Z)/14:1(9Z)) & $\uparrow \uparrow$ & Glycerophospholipids & Wang et al., 2015 & LC-MS \\
\hline LysoPC (18:1(9Z)) & $\uparrow \uparrow$ & Glycerophospholipids & Wang et al., 2015 & LC-MS \\
\hline Cer (d18:0/20:0) & $\downarrow$ & Sphingolipids & Wang et al., 2015 & LC-MS \\
\hline Phytosphingosine & $\downarrow$ & Sphingolipids & Wang et al., 2015 & LC-MS \\
\hline Glycocholic acid & $\downarrow$ & Steroids & Wang et al., 2015 & LC-MS \\
\hline $\begin{array}{l}\text { Chenodeoxycholic acid } \\
\text { 3-sulphate }\end{array}$ & $\downarrow$ & Steroids & Wang et al., 2015 & LC-MS \\
\hline 3-Oxo-4,6-choladienoic acid & $\downarrow$ & Steroids & Wang et al., 2015 & LC-MS \\
\hline Cortolone-3-glucuronide & $\uparrow$ & Steroids & Wang et al., 2015 & LC-MS \\
\hline $11 \alpha$-Hydroxyprogesterone & $\uparrow$ & Steroids & Wang et al., 2015 & LC-MS \\
\hline Testosterone glucuronide & $\uparrow$ & Steroids & Wang et al., 2015 & LC-MS \\
\hline Tetrahydroaldosterone-3-gluct & onide & Steroids & Wang et al., 2015 & LC-MS \\
\hline
\end{tabular}


Table 2. Cont.

\begin{tabular}{|c|c|c|c|c|}
\hline Metabolites & PCOS vs. Control & Metabolic Pathways & Studies & Techniques \\
\hline Dehydroepiandrosterone & $\uparrow$ & Androgen metabolism & Dhayat et al., 2018 & GC-MS \\
\hline $16 \alpha-\mathrm{OH}$-dehydroepiandrosterone & $\uparrow$ & Androgen metabolism & Dhayat et al., 2018 & GC-MS \\
\hline Androstenediol & $\uparrow$ & Androgen metabolism & Dhayat et al., 2018 & GC-MS \\
\hline Testosterone & $\uparrow$ & Androgen metabolism & Dhayat et al., 2018 & GC-MS \\
\hline $5 \alpha$-DH-testosterone & $\uparrow$ & Androgen metabolism & Dhayat et al., 2018 & GC-MS \\
\hline Androstanediol & $\uparrow$ & Androgen metabolism & Dhayat et al., 2018 & GC-MS \\
\hline Androsterone & $\uparrow$ & Androgen metabolism & Dhayat et al., 2018 & GC-MS \\
\hline $11 \beta$-OH-androsterone & $\uparrow$ & Androgen metabolism & Dhayat et al., 2018 & GC-MS \\
\hline Etiocholanolone & $\uparrow$ & Androgen metabolism & Dhayat et al., 2018 & GC-MS \\
\hline Estriol & $\downarrow$ & Estrogen metabolism & Dhayat et al., 2018 & GC-MS \\
\hline Suberic acid & $\uparrow$ & Fatty acid metabolism & Zou et al., 2018 & GC-MS \\
\hline 3,4,5-hydroxyvaleric acid & $\uparrow$ & Fatty acid metabolism & Zou et al., 2018 & GC-MS \\
\hline $\begin{array}{c}\text { (R)-3-Hydroxy- } \\
\text { hexadecanoic acid }\end{array}$ & $\downarrow$ & Fatty acid metabolism & Wang et al., 2015 & LC-MS \\
\hline 6-Keto-decanoylcarnitine & $\downarrow$ & Fatty acid esters & Wang et al., 2015 & LC-MS \\
\hline Tiglylcarnitine & $\uparrow$ & Fatty acid esters & Wang et al., 2015 & LC-MS \\
\hline Butyrylcarnitine & $\uparrow \uparrow$ & Fatty acid esters & Wang et al., 2015 & LC-MS \\
\hline 4-hydroxyphenylacetic acid & $\uparrow \uparrow$ & Tyrosine metabolism & Zou et al., 2018 & GC-MS \\
\hline Capryloylglycine & $\downarrow$ & $\begin{array}{l}\text { Amino acid } \\
\text { metabolism }\end{array}$ & Wang et al., 2015 & LC-MS \\
\hline $\mathrm{N}$-(7-Isocucurbinoyl)isoleucine & $\uparrow$ & $\begin{array}{l}\text { Amino acid } \\
\text { metabolism }\end{array}$ & Wang et al., 2015 & LC-MS \\
\hline Aspartylglycosamine & $\uparrow$ & $\begin{array}{l}\text { Amino acid } \\
\text { metabolism }\end{array}$ & Wang et al., 2015 & LC-MS \\
\hline$\alpha$-ketoglutarate & $\uparrow$ & $\begin{array}{l}\text { Amino acid } \\
\text { metabolism }\end{array}$ & Zou et al., 2018 & GC-MS \\
\hline Threonine & $\uparrow$ & $\begin{array}{l}\text { Amino acid } \\
\text { metabolism }\end{array}$ & Zou et al., 2018 & GC-MS \\
\hline Serine & $\uparrow$ & $\begin{array}{l}\text { Amino acid } \\
\text { metabolism }\end{array}$ & Zou et al., 2018 & GC-MS \\
\hline Glycine & $\uparrow$ & $\begin{array}{l}\text { Amino acid } \\
\text { metabolism }\end{array}$ & Zou et al., 2018 & GC-MS \\
\hline 5-Oxoproline & $\uparrow$ & $\begin{array}{l}\text { Amino acid } \\
\text { metabolism }\end{array}$ & Zou et al., 2018 & GC-MS \\
\hline Benzoylglycine & $\uparrow$ & $\begin{array}{l}\text { Amino acid } \\
\text { metabolism }\end{array}$ & Zou et al., 2018 & GC-MS \\
\hline Indoleacetyl glutamine & $\downarrow$ & Aromatic Amino acids & Wang et al., 2015 & LC-MS \\
\hline Flazine methyl ether & $\downarrow$ & Aromatic Amino acids & Wang et al., 2015 & LC-MS \\
\hline Succinyladenosine & $\uparrow$ & Aromatic Amino acids & Wang et al., 2015 & LC-MS \\
\hline Thyronine & $\uparrow$ & Aromatic Amino acids & Wang et al., 2015 & LC-MS \\
\hline Gamma-glutamyl-leucine & $\downarrow$ & Peptides & Wang et al., 2015 & LC-MS \\
\hline Tryptophyl-proline & $\downarrow$ & Peptides & Wang et al., 2015 & LC-MS \\
\hline Methionyl-phenylalanine & $\uparrow$ & Peptides & Wang et al., 2015 & LC-MS \\
\hline Phenylalanyl-histidine & $\uparrow$ & Peptides & Wang et al., 2015 & LC-MS \\
\hline Arginyl-valiney & $\uparrow$ & Peptides & Wang et al., 2015 & LC-MS \\
\hline
\end{tabular}


Table 2. Cont.

\begin{tabular}{|c|c|c|c|c|}
\hline Metabolites & PCOS vs. Control & Metabolic Pathways & Studies & Techniques \\
\hline Threoninyl-lysine & $\uparrow$ & Peptides & Wang et al., 2015 & LC-MS \\
\hline Tryptophyl-arginine & $\uparrow$ & Peptides & Wang et al., 2015 & LC-MS \\
\hline Tyrosyl-leucine & $\uparrow$ & Peptides & Wang et al., 2015 & LC-MS \\
\hline Tryptophyl-valine & $\uparrow$ & Peptides & Wang et al., 2015 & LC-MS \\
\hline Cis-aconitic acid & $\uparrow$ & CTA metabolism & Zou et al., 2018 & GC-MS \\
\hline $\begin{array}{c}\text { 3-Hydroxy-3- } \\
\text { Methylglutaric acid }\end{array}$ & $\uparrow$ & Energy metabolism & Zou et al., 2018 & GC-MS \\
\hline 2-Hydroxyglutaric acid & $\uparrow$ & Energy metabolism & Zou et al., 2018 & GC-MS \\
\hline Threonic acid & $\uparrow$ & $\begin{array}{l}\text { Sugar acids } \\
\text { metabolism }\end{array}$ & Zou et al., 2018 & GC-MS \\
\hline Inosine & $\uparrow$ & Purine metabolism & Zou et al., 2018 & GC-MS \\
\hline 2,3,4-Hydroxybutyric acid & $\uparrow$ & Energy metabolism & Zou et al., 2018 & GC-MS \\
\hline 3,4-Hydroxybutyric acid & $\uparrow$ & Energy metabolism & Zou et al., 2018 & GC-MS \\
\hline 4-Hydroxybutyric acid & $\uparrow$ & Energy metabolism & Zou et al., 2018 & GC-MS \\
\hline 2-Hydroxyisobutyric acid & $\uparrow$ & Energy metabolism & Zou et al., 2018 & GC-MS \\
\hline Uracil & $\uparrow$ & $\begin{array}{l}\text { Pyrimidine } \\
\text { metabolism }\end{array}$ & Zou et al., 2018 & GC-MS \\
\hline Glyceryl acid & $\uparrow$ & $\begin{array}{l}\text { Hydroxy acid } \\
\text { metabolism }\end{array}$ & Zou et al., 2018 & GC-MS \\
\hline Glycolic acid & $\uparrow$ & $\begin{array}{l}\text { Hydroxy acid } \\
\text { metabolism }\end{array}$ & Zou et al., 2018 & GC-MS \\
\hline 2-Hydroxyisobutyric acid & $\uparrow \uparrow$ & Energy metabolism & Zou et al., 2018 & GC-MS \\
\hline Succinic acid & $\downarrow$ & Glucose metabolism & Zou et al., 2018 & GC-MS \\
\hline Benzophenone & $\uparrow$ & Acetophenones & Wang et al., 2015 & LC-MS \\
\hline 5'-Carboxy- $\gamma$-chromanol & $\downarrow$ & Benzopyrans & Wang et al., 2015 & LC-MS \\
\hline $5^{\prime}$-Carboxy- $\alpha$-chromanol & $\downarrow$ & Benzopyrans & Wang et al., 2015 & LC-MS \\
\hline 9'-Carboxy- $\alpha$-chromanol & $\downarrow$ & Benzopyrans & Wang et al., 2015 & LC-MS \\
\hline $11^{\prime}$-Carboxy- $\alpha$-tocotrienol & $\downarrow$ & Benzopyrans & Wang et al., 2015 & LC-MS \\
\hline FMNH2 & $\uparrow$ & Pteridines & Wang et al., 2015 & LC-MS \\
\hline Urobilin & $\downarrow$ & Tetrapyrroles & Wang et al., 2015 & LC-MS \\
\hline Mesobilirubinogen & $\downarrow$ & Tetrapyrroles & Wang et al., 2015 & LC-MS \\
\hline Harderoporphyrinogen & $\downarrow$ & Tetrapyrroles & Wang et al., 2015 & LC-MS \\
\hline $\begin{array}{c}\text { MG } \\
(18: 4(6 Z, 9 Z, 12 Z, 15 Z) / 0: 0 / 0: 0)\end{array}$ & $\uparrow$ & Lineolic acids & Wang et al., 2015 & LC-MS \\
\hline Hydroxyvalerylcarnitine & $\uparrow$ & Alkylamines & Wang et al., 2015 & LC-MS \\
\hline Labadoside & $\uparrow$ & Glycosides & Wang et al., 2015 & LC-MS \\
\hline $\begin{array}{l}\text { Dihydrocaffeic acid } \\
\text { 3-O-glucuronide }\end{array}$ & $\downarrow$ & Sugar acids & Wang et al., 2015 & LC-MS \\
\hline $\begin{array}{l}\text { Dihydroferulic acid } \\
\text { 4-O-glucuronide }\end{array}$ & $\downarrow$ & Sugar acids & Wang et al., 2015 & LC-MS \\
\hline $\begin{array}{c}\text { 5-Hydroxy-6- } \\
\text { methoxyindole glucuronide }\end{array}$ & $\uparrow$ & Sugar acids & Wang et al., 2015 & LC-MS \\
\hline p-Cresol glucuronide & $\downarrow$ & Sugar acids & Wang et al., 2015 & LC-MS \\
\hline $6 \beta-\mathrm{OH}$-cortisol & $\uparrow$ & $\begin{array}{l}\text { Glucocorticoid } \\
\text { metabolism }\end{array}$ & Dhayat et al., 2018 & GC-MS \\
\hline
\end{tabular}


Table 2. Cont.

\begin{tabular}{ccccc}
\hline Metabolites & PCOS vs. Control & Metabolic Pathways & Studies & Techniques \\
\hline 18-OH-cortisol & $\uparrow$ & $\begin{array}{c}\text { Glucocorticoid } \\
\text { metabolism }\end{array}$ & Dhayat et al., 2018 & GC-MS \\
\hline TH-cortisol & $\uparrow$ & $\begin{array}{c}\text { Glucocorticoid } \\
\text { metabolism }\end{array}$ & Dhayat et al., 2018 & GC-MS \\
\hline $11 \beta$-OH-etiocholanolone & $\uparrow$ & $\begin{array}{c}\text { Glucocorticoid } \\
\text { metabolism }\end{array}$ & Dhayat et al., 2018 & GC-MS \\
\hline TH-cortisone & $\uparrow$ & $\begin{array}{c}\text { Glucocorticoid } \\
\text { metabolism }\end{array}$ & Dhayat et al., 2018 & GC-MS \\
\hline
\end{tabular}

TG = triglyceride, $\mathrm{DG}=$ diglyceride, $\mathrm{PC}=$ phosphatidylcholine, $($ LysoPC $)=$ lysophosphatidylcholine,

LPA $=$ lysophosphatidic acid, FMNH2 = reduced flavin mononucleotide; $\uparrow$ up-regulation; $\downarrow$ down-regulation.

\subsection{Metabolomic Profile of Follicular Fluid Samples}

Follicular fluid (FF) is an alternative and a useful biological matrix to study the potential mechanism of PCOS pathophysiology. FF is the product of plasma modified by the secretory activity of the granulosa and theca cells [42]. This matrix is collected from women with PCOS undergoing in vitro fertilization. FF contains metabolites essential for oocyte growth and maturation. The analysis of the metabolic derangements in FF samples from women with PCOS allows to understand pathological changes and also disclose the metabolites that could potentially disturb normal oocyte growth [43].

We reviewed a few metabolomic studies that analysed FF from women with PCOS and compared them with healthy controls [44-48]. They were performed with the use of different metabolomic techniques. As can be observed, the concentration of metabolites involved in the TCA cycle, as well as $\alpha$-keto acids, are relatively higher in samples obtained from women with PCOS in comparison with the control subjects. The contrary trend was shown for acylcarnitines. For all of the determined metabolites belonging to acylcarnitines, the decreased level was determined for samples obtained from PCOS patients. However, there are a few metabolic pathways where the trend is not similar among the pathway, but is specific for individual subgroups of compounds. For example, as was reported by Liu et al. (2018), the decreased level of PC was observed in PCOS patients, while the contrary trend (up-regulation) was shown for Lyso PC [46]. In the case of fatty acyls, the general direction demonstrates down-regulation. However, Liu et al. (2018) reported an increased level of 1-Hydroxy-2,12,15-heneicosatrien-4-one in PCOS patients in comparison with the control subjects. The diversity was also observed in the case of AAs metabolism. For example, increased level of phenylalanine, valine, and isoleucine was observed, while a decreased amount of alanine, glutamine, and tyrosine was reported. The results are presented in Table 3.

Table 3. The most significant changes in FF metabolites in women with PCOS in comparison with the control subjects.

\begin{tabular}{ccccc}
\hline Metabolites & PCOS vs. Control & Metabolic Pathways & Studies & Techniques \\
\hline Paxilline & $\downarrow$ & Naphthopyrans & Liu et al., 2018 & LC-MS \\
\hline PC (o-22:0/20:4(8Z,11Z,14Z,17Z)) & $\downarrow$ & Glycerophospholipid & Liu et al., 2018 & LC-MS \\
\hline PC & $\downarrow$ & Glycerophospholipid & Liu et al., 2018 & LC-MS \\
$($ o22:0/22:6(4Z,7Z,10Z,13Z,16Z,19Z)) & $\uparrow$ & Glycerophospholipid & Liu et al., 2018 & LC-MS \\
\hline LysoPC $(16: 1(9 Z))$ & Glycerophospholipid & $\begin{array}{l}\text { Liu et al., 2018 } \\
\text { Sun et al., 2019 }\end{array}$ & LC-MS \\
\hline LysoPC (16:0) & $\uparrow$ & Glycerophospholipid & Sun et al., 2019 & LC-MS \\
\hline LysoPC (14:0) & $\uparrow$ & Glycerophospholipid & Sun et al., 2019 & LC-MS \\
\hline LysoPC (18:0) & $\uparrow$ & & &
\end{tabular}


Table 3. Cont

\begin{tabular}{|c|c|c|c|c|}
\hline Metabolites & PCOS vs. Control & Metabolic Pathways & Studies & Techniques \\
\hline LysoPC (20:4(8Z,11Z,14Z,17Z)) & $\uparrow$ & Glycerophospholipid & Liu et al., 2018 & LC-MS \\
\hline PGP (16:0/20:4(5Z,8Z,11Z,14Z) & $\downarrow$ & Glycerophospholipid & Liu et al., 2018 & LC-MS \\
\hline Glycerophosphocholine & $\uparrow$ & Glycerophospholipid & Chen et al., 2020 & LC-MS \\
\hline Ceramide (d18:0/16:0) & $\downarrow$ & Sphingolipids & Liu et al., 2018 & LC-MS \\
\hline Ceramide (d18:0/24:0) & $\downarrow$ & Sphingolipids & Liu et al., 2018 & LC-MS \\
\hline $\begin{array}{l}\text { Galabiosylceramide } \\
\text { (d18:1/24:1(15Z)) }\end{array}$ & $\downarrow$ & Sphingolipids & Liu et al., 2018 & LC-MS \\
\hline Tetrahexosylceramide (d18:1/24:0) & $\downarrow$ & Sphingolipids & Liu et al., 2018 & LC-MS \\
\hline $7 \beta$-Hydroxycholesterol & $\downarrow$ & Lipid metabolism & Chen et al., 2020 & LC-MS \\
\hline Malyl-CoA & $\downarrow$ & Fatty Acyls & Liu et al., 2018 & LC-MS \\
\hline $\begin{array}{l}\text { 1-Hydroxy-2,12,15- } \\
\text { heneicosatrien-4-one }\end{array}$ & $\uparrow$ & Fatty Acyls & Liu et al., 2018 & LC-MS \\
\hline 16-hydroxypalmitic acid & $\downarrow$ & Fatty Acyls & Liu et al., 2018 & LC-MS \\
\hline Tridecanol & $\downarrow$ & Fatty Acyls & Liu et al., 2018 & LC-MS \\
\hline Carnitine & $\uparrow$ & $\begin{array}{l}\text { Fatty acids } \\
\text { metabolism }\end{array}$ & Chen et al., 2020 & LC-MS \\
\hline $\begin{array}{l}\text { 4-Hydroxy-3-(16-methylheptadecyl)- } \\
\text { 2H-pyran-2-one }\end{array}$ & $\downarrow$ & Pyrans & Liu et al., 2018 & LC-MS \\
\hline Anandamide & $\downarrow$ & $\begin{array}{l}\text { Organonitrogen } \\
\text { compounds }\end{array}$ & Liu et al., 2018 & LC-MS \\
\hline Indan-1-ol & $\uparrow$ & Indanes & Liu et al., 2018 & LC-MS \\
\hline $\begin{array}{c}\text { 2-p-Tolyl-1-propene, } \\
\text { p-Mentha-1,3,5,8-tetraene }\end{array}$ & $\uparrow$ & Phenylpropenes & Liu et al., 2018 & LC-MS \\
\hline$\beta$-Ionol & $\downarrow$ & Sesquiterpenoids & Liu et al., 2018 & LC-MS \\
\hline Androstenol & $\downarrow$ & Androstane steroids & Liu et al., 2018 & LC-MS \\
\hline $\begin{array}{c}\text { (3R, 6' Z)-3,4-Dihydro-8-hydroxy-3- } \\
\text { (6-pentadecenyl)-1H- } \\
\text { 2-benzopyran-1-one }\end{array}$ & $\downarrow$ & Benzopyrans & Liu et al., 2018 & LC-MS \\
\hline 6-Tridecylsalicylic acid & $\downarrow$ & $\begin{array}{l}\text { Benzoic acids } \\
\text { and derivatives }\end{array}$ & Liu et al., 2018 & LC-MS \\
\hline 2,3-dihydroxypropyl dodecanoate & $\uparrow$ & Glycerol metabolism & Chen et al., 2020 & LC-MS \\
\hline Methylmalonic acid & $\downarrow$ & Carboxylic acids & Liu et al., 2018 & LC-MS \\
\hline Lysyl-Valine & $\downarrow$ & $\begin{array}{l}\text { Carboxylic acids } \\
\text { and derivatives }\end{array}$ & Liu et al., 2018 & LC-MS \\
\hline Prolyl-Methionine & $\downarrow$ & $\begin{array}{l}\text { Carboxylic acids } \\
\text { and derivative }\end{array}$ & Liu et al., 2018 & LC-MS \\
\hline VPGPR Enterostatin & $\downarrow$ & $\begin{array}{l}\text { Carboxylic acids } \\
\text { and derivative }\end{array}$ & Liu et al., 2018 & LC-MS \\
\hline 1H-Indol-3-ylacetyl-myo-inositol & $\downarrow$ & $\begin{array}{c}\text { Indoles } \\
\text { and derivatives }\end{array}$ & Liu et al., 2018 & LC-MS \\
\hline 1-Pentadecene & $\downarrow$ & $\begin{array}{l}\text { Unsaturated } \\
\text { hydrocarbons }\end{array}$ & Liu et al., 2018 & LC-MS \\
\hline Lithocholic acid glycine conjugate & $\downarrow$ & Non classified & Liu et al., 2018 & LC-MS \\
\hline Lactate & $\begin{array}{l}\downarrow \\
\downarrow\end{array}$ & $\begin{array}{l}\text { Gluconeogenesis/ } \\
\text { Glycolysis }\end{array}$ & $\begin{array}{l}\text { Zhang et al., } 2017 \\
\text { Liu et al., } 2018\end{array}$ & $\begin{array}{l}\text { NMR } \\
\text { GC-MS }\end{array}$ \\
\hline Glyceraldehyde & $\uparrow$ & $\begin{array}{c}\text { Gluconeogenesis/ } \\
\text { Glycolysis }\end{array}$ & Chen et al., 2020 & LC-MS \\
\hline
\end{tabular}


Table 3. Cont.

\begin{tabular}{|c|c|c|c|c|}
\hline Metabolites & PCOS vs. Control & Metabolic Pathways & Studies & Techniques \\
\hline Pyruvate & $\begin{array}{l}\downarrow \\
\uparrow\end{array}$ & Glucose glycolysis & $\begin{array}{l}\text { Zhang et al., } 2017 \\
\text { Zhao et al., } 2015\end{array}$ & $\begin{array}{l}\text { NMR } \\
\text { GC-MS }\end{array}$ \\
\hline Valine & $\uparrow$ & $\begin{array}{l}\text { Amino acid } \\
\text { metabolism }\end{array}$ & Zhao et al., 2015 & MS/MS \\
\hline Isoleucine & $\uparrow$ & $\begin{array}{l}\text { Amino acid } \\
\text { metabolism }\end{array}$ & Zhao et al., 2015 & MS/MS \\
\hline Leucine & $\uparrow$ & $\begin{array}{l}\text { Amino acid } \\
\text { metabolism }\end{array}$ & $\begin{array}{l}\text { Zhao et al., } 2015 \\
\text { Sun et al., } 2019\end{array}$ & $\begin{array}{l}\text { MS/MS } \\
\text { LC-MS }\end{array}$ \\
\hline Alanine & $\downarrow$ & $\begin{array}{l}\text { Amino acid } \\
\text { metabolism }\end{array}$ & Zhang et al., 2017 & NMR \\
\hline Glutamine & $\downarrow$ & $\begin{array}{l}\text { Amino acid } \\
\text { metabolism }\end{array}$ & Zhang et al., 2017 & NMR \\
\hline Tyrosine & $\downarrow$ & $\begin{array}{l}\text { Amino acid } \\
\text { metabolism }\end{array}$ & Zhang et al., 2017 & NMR \\
\hline Phenylalanine & $\uparrow$ & $\begin{array}{l}\text { Amino acid } \\
\text { metabolism }\end{array}$ & Sun et al., 2019 & LC-MS \\
\hline D-Glutamic acid & $\uparrow$ & $\begin{array}{l}\text { Amino acid } \\
\text { metabolism }\end{array}$ & Chen et al., 2020 & LC-MS \\
\hline Ferulic acid & $\uparrow$ & $\begin{array}{l}\text { Amino acid } \\
\text { metabolism }\end{array}$ & Chen et al., 2020 & LC-MS \\
\hline Salicylic acid & $\uparrow$ & $\begin{array}{l}\text { Amino acid } \\
\text { metabolism }\end{array}$ & Chen et al., 2020 & LC-MS \\
\hline Lysine & $\uparrow$ & $\begin{array}{l}\text { Amino acid } \\
\text { metabolism }\end{array}$ & Chen et al., 2020 & LC-MS \\
\hline 3-Methylhistidine & $\uparrow$ & $\begin{array}{l}\text { Amino acid } \\
\text { metabolism }\end{array}$ & Chen et al., 2020 & LC-MS \\
\hline$\alpha$-Keto- $\beta$-methylvalerate & $\uparrow$ & Alpha-keto acids & Zhao et al., 2015 & GC-MS \\
\hline$\alpha$-Ketoisovalerate & $\uparrow$ & Alpha-keto acids & Zhao et al., 2015 & GC-MS \\
\hline$\alpha$-Ketoisocaproate & $\uparrow$ & Alpha-keto acids & Zhao et al., 2015 & GC-MS \\
\hline Hexanoyl (C6) & $\downarrow$ & Acylcarnitines & Zhao et al., 2015 & MS/MS \\
\hline Malonyl (C3DC) & $\downarrow$ & Acylcarnitines & Zhao et al., 2015 & MS/MS \\
\hline Hydroxyisovaleryl (C5OH) & $\downarrow$ & Acylcarnitines & Zhao et al., 2015 & MS/MS \\
\hline Octenoyl (C8:1) & $\downarrow$ & Acylcarnitines & Zhao et al., 2015 & MS/MS \\
\hline Adipyl (C6DC) & $\downarrow$ & Acylcarnitines & Zhao et al., 2015 & MS/MS \\
\hline$\beta$-Hydroxybutyrate & $\uparrow$ & Ketones & Zhao et al., 2015 & GC-MS \\
\hline Succinate & $\uparrow$ & TCA cycle metabolites & Zhao et al., 2015 & GC-MS \\
\hline Malate & $\uparrow$ & TCA cycle metabolites & Zhao et al., 2015 & GC-MS \\
\hline Oxaloacetate & $\uparrow$ & TCA cycle metabolites & Zhao et al., 2015 & GC-MS \\
\hline Cis-aconitate & $\downarrow$ & TCA cycle metabolites & Zhao et al., 2015 & GC-MS \\
\hline Acetate & $\uparrow$ & TCA cycle metabolites & Zhang et al., 2017 & NMR \\
\hline Acetoacetate & $\uparrow$ & TCA cycle metabolites & Zhang et al., 2017 & NMR \\
\hline 3-Hyroxybutyrate & $\uparrow$ & TCA cycle metabolites & Zhang et al., 2017 & NMR \\
\hline$N$-Methylnicotinamide & $\downarrow$ & $\begin{array}{l}\text { Metabolites of NAD } \\
\text { catabolism }\end{array}$ & Zhao et al., 2015 & LC-MS/MS \\
\hline $\begin{array}{l}\text { N1-Methyl-2-pyridone-5- } \\
\text { carboxamide (2PY) }\end{array}$ & $\downarrow$ & $\begin{array}{l}\text { Metabolites of NAD } \\
\text { catabolism }\end{array}$ & Zhao et al., 2015 & LC-MS/MS \\
\hline $\begin{array}{l}\text { N1-Methyl-4-pyridone-3- } \\
\text { carboxamide (4PY) }\end{array}$ & $\downarrow$ & $\begin{array}{l}\text { Metabolites of NAD } \\
\text { catabolism }\end{array}$ & Zhao et al., 2015 & LC-MS/MS \\
\hline
\end{tabular}


Table 3. Cont.

\begin{tabular}{|c|c|c|c|c|}
\hline Metabolites & PCOS vs. Control & Metabolic Pathways & Studies & Techniques \\
\hline Deoxycorticosterone & $\uparrow$ & Steroid metabolism & Sun et al., 2019 & LC-MS \\
\hline Pregnenolone & $\downarrow$ & Steroid metabolism & Chen et al., 2020 & LC-MS \\
\hline 17-Hydroxyprogesterone & $\downarrow$ & Steroid metabolism & Chen et al., 2020 & LC-MS \\
\hline 3-Hydroxynonanoyl carnitine & $\uparrow$ & Fatty acid metabolism & Sun et al., 2019 & LC-MS \\
\hline Eicosapentaenoic acid & $\uparrow$ & Fatty acid metabolism & Sun et al., 2019 & LC-MS \\
\hline Phytosphingosine & $\downarrow$ & $\begin{array}{l}\text { Sphingolipid } \\
\text { metabolism }\end{array}$ & Sun et al., 2019 & LC-MS \\
\hline $\mathrm{N}$-acetylneuraminic acid & $\uparrow$ & Sialic acid metabolism & Chen et al., 2020 & LC-MS \\
\hline Pyridoxal 5'-phosphate & $\uparrow$ & $\begin{array}{l}\text { Vitamin B6 } \\
\text { metabolism }\end{array}$ & Chen et al., 2020 & LC-MS \\
\hline Purine & $\uparrow$ & Purines metabolism & Chen et al., 2020 & LC-MS \\
\hline 1,3-Dimethyluracil & $\uparrow$ & Purines metabolism & Chen et al., 2020 & LC-MS \\
\hline Oxalic acid & $\uparrow$ & $\begin{array}{l}\text { Glyoxylic acid } \\
\text { metabolism }\end{array}$ & Chen et al., 2020 & LC-MS \\
\hline Phenylglyoxylic acid & $\uparrow$ & $\begin{array}{l}\text { Glyoxylic acid } \\
\text { metabolism }\end{array}$ & Chen et al., 2020 & LC-MS \\
\hline
\end{tabular}

$\mathrm{PC}=$ phosphatidylcholine $($ LysoPC $)$ lysophosphatidylcholine, $\mathrm{PGP}$ = glycerol-3-phosphate, $\mathrm{CoA}=$ coenzyme $\mathrm{A}$; $\uparrow$ up-regulation; $\downarrow$ down-regulation.

\section{Discussion}

The summary of all the metabolites detected in three different biological matrices gives us a complementary overview of the metabolomic profile of women with PCOS and allows us to look for a correlation between altered levels of metabolites from different biochemical pathways.

Lipids are the largest group of molecules whose metabolism is deranged in women with PCOS. They are involved in various metabolic pathways, such as steroid hormone biosynthesis, sphingolipid, and fatty acids metabolisms like oxidation or amide metabolism. Phospholipids, which take part in multiple biological pathways, are down-regulated in PCOS. There are few studies confirming the decreased levels of sphinganine, LPE, especially LPE (22:5) and LPC, mainly LPC (18:2) in women with PCOS [27,28,34]. LPCs are involved in glucose metabolism, and low levels of LPC (18:2) correlate with IR and increased risk of T2DM. Thus, this would be in accordance with the observation that women with PCOS are more prone to these metabolic alterations [34]. Hauola et al. (2015) noticed that the differences in the lipid profiles between PCOS and healthy women correlate with the phase of the menstrual cycle. The most significant changes were detected between samples collected from healthy women during the luteal phase of the menstrual cycle and women with PCOS [35]. Among sphingolipids, phytosphingosine (PHS), which stimulates the transcriptional activity of the peroxisome proliferator-activated receptor $\gamma(\operatorname{PPAR} \gamma)$, is downregulated in women with PCOS. According to the literature, PPARs are ligand-dependent transcription factors that regulate the expression of numerous genes associated with the metabolism of carbohydrates, lipids, and proteins [30]. Dysfunction of these receptors may also contribute to the increased risk of MetS and T2DM. The latest lipidomic studies indicate that elevated levels of DG and cholesterol ester, and lower levels of LysoPC correlate with IR, irrespective of a person being overweight or obese [49].

Szczuko et al. (2017) analysed plasma fatty acids in women with PCOS. Their results showed that the levels of all free fatty acids were lower in women with PCOS; however, the concentration of nervonic acid was several times (almost 330 times) higher than in the control subjects. This level of nervonic acid was observed in two analysed groups of women, namely women with PCOS with a biochemical indication of hyperandrogenism and women with normal androgen levels. Poly-unsaturated fatty acids (PUFAs), the precursors of eicosanoids, were significantly increased in women with PCOS compared with the control subjects, which may be due to the presence of low-grade systemic inflammation. 
In recent years, some studies reported that this process could be stimulated by the pro-inflammatory interleukin-1 (IL-1), which is overexpressed in women with PCOS [50].

One of the detected metabolites, which may be considered as a potential biomarker of PCOS, is DHEA. Excess levels of DHEA are mostly detected in the metabolome of women with PCOS [29, 30,33,34]. Serum concentrations of DHEA-S are also evaluated in clinical practice for the evaluation of biochemical hyperandrogenism in women. Besides, 19-oxotestosterone levels are also elevated in women with POCS. This could be due to the higher activity of aromatase, which catalyses the formation of C18 estrogens from C19 androgens [30]. Zhao et al. (2014) showed that, apart from elevated DHEA levels in women with PCOS, there is a significant increase of DHTS and ANDS, which points to the exaggerated androgen synthesis [29]. Dhayat et al. (2018) reported that in women with PCOS, there is an alternative pathway of 11-oxygenated androgen production. They also reported that four steroids such as androstanediol, estriol, 20- $\beta$-dihydrocortisone, and cortisol are found as potential markers of PCOS [41].

Lipidomic analysis was also the main goal of the study conducted by Vonica et al. (2019). The authors reported that alterations in acylglycerols, PGs and LTs, phosphocholines, and carnitine metabolites occur in women with PCOS. As a result, cholestane-5 $\alpha$ (18:1/0:0), triacylglycerol (18:2/18:2/0-18:0), cholestane-3 $3,5 \alpha, 6 \beta$-triol (18:0/0:0) were found as the crucial metabolites to identify women with PCOS from the controls. Serum levels of these metabolites were decreased. Moreover, elevated acylcarnitine: 2-hydroxylauroylcarnitine with decreased phosphocholines metabolites (18:1/18:4, 18:3/18:2) were also observed. The authors assumed that this alteration might be linked to the lipid peroxidation. Levels of some of the TG (18:2/18:2/0-18:0) and DG species (18:1/20:0/0:0, 18:0/0:0/20:1, 22:4n6/0:0/18:4n3, 18:3/0:0/22:5, 20:0/0:0/20:0, 18:1/24:0/0:0, 18:0/0:0/24:1) were also elevated in women with PCOS in comparison with the control subjects [37]. This can be explained by a higher BMI and increased intra-abdominal fat deposition, which is also a hallmark of PCOS. Dyslipidemia, which occurs in PCOS is characterized by the increased concentrations of total cholesterol (TCh), low density lipoproteins (LDL), very-low density lipoproteins (VLDL), and TGs coupled with decreased high-density lipoproteins (HDL) and HDL-cholesterol. During intestinal absorption, TGs are degraded to fatty acids. After that, they are again resynthesized and transported by the chylomicrons through the bloodstream to the adipose and muscle tissue, where their degradation into free fatty acids and monoacylglycerol takes place. Elevated levels of TGs in PCOS women may be caused by a high fat intake or reduced fat energy consumption at night. It was confirmed that in women with PCOS, the reduced ability to switch to lipid oxidation during fasting also occurs at night [37].

$7 \beta$-Hydroxycholesterol is another lipid metabolite that could also be a potential biomarker of PCOS. It highly correlates with a PCOS diagnosis. This oxysterol is found as a metabolic intermediate or the end-product of cholesterol metabolism. Due to its bioactivity, it could induce oxidative stress and disturb the metabolism of fatty acids. Chen et al. (2020) showed that elevated levels of $7 \beta$-hydroxycholesterol measured in the FF by the induction of the oxidative stress may disturb the growth of oocytes in PCOS [48].

There also are some specific alterations of the bile acid metabolism. These abnormalities and dysfunction of fat absorption in women with PCOS is due to the decreased level of glycocholic acid, which was reported by Zhao et al. (2014) [29] and confirmed by Jia et al. (2019) [34].

The metabolism of prostaglandins and carnitine also seems to be deranged in women with PCOS. Vonica et al. (2019) reported an increased level of the prostaglandin (PG) E2 pathway and oxo-leukotrienes (LT) known to play a pivotal role in inflammation [37]. Dong et al. (2015) showed an increase of prostaglandin F2a (FPG-2a) and a decrease of L-carnitine in women with PCOS [30]. Elevated levels of FPG-2a may also be related to the presence of a low-grade systemic inflammation in PCOS. In turn, L-carnitine plays an essential role in fatty acid metabolism as well as their transport across the mitochondrial membrane to the mitochondrial matrix where $\beta$-oxidation of fatty acids (FAs) occurs. Decreased levels of L-carnitine may point to the impairment of these processes in PCOS. It could result in an accumulation of the FAs in the cytosol. It is noticed that 
L-carnitine supplementation may improve oocyte quality and therefore, may have a positive effect on fertility [30]. Carnitine is also involved in stabilizing acetylCoA and coenzyme A levels and plays an essential role during fetal maturation [34]. Elevated levels of acetate found by Whigham et al. (2014) and RoyChodhury et al. (2016) also indicate reduced FAs oxidation in women with PCOS [28].

Impaired carbohydrate metabolism is a hallmark of PCOS. Elevated serum lactate and reduced level of glucose determined in plasma samples suggest alterations in glucose metabolism accompanied with elevated glycolytic activity associated with the TCA cycle impairment. Whigham et al. (2014) suggested that in women with PCOS, some AAs are utilized as a source of alternative energy metabolism [24]. Alanine is an important amino acid in the process of gluconeogenesis. Serum alanine-amino transaminase (ALT) activity is elevated in women with PCOS, which usually is the result of NAFLD and lead to increased alanine transformation [27]. According to the literature, ALT is involved in urea cycle and AAs metabolism, where alanine may be converted to pyruvate by donating an amine group and enter the TCA cycle as well as be formed from pyruvate by accepting an amine group, respectively. Serum levels of glucogenic AAs acids such as valine, leucine, and threonine, which may also enter the TCA cycle or be involved in the process of gluconeogenesis were also significantly elevated in women with PCOS. The higher number of growing antral follicles in PCOS utilizes more energy. For this purpose, carbohydrate metabolism may not be sufficient and may thus lead to the utilization of other energy substrates like glutamine, glutamate, or 3-hydroxybutyric acid. Whigham et al. (2014) pointed out that the TCA cycle and glucose metabolism are the major pathways deranged in PCOS [28]. As reported, glucose metabolism can be carried out via alternative pathways such as glycolysis or pentose phosphate pathways and lead to an increased FAs synthesis, which may explain the observed increased FAs accumulation in the adipocytes (Figure 2).

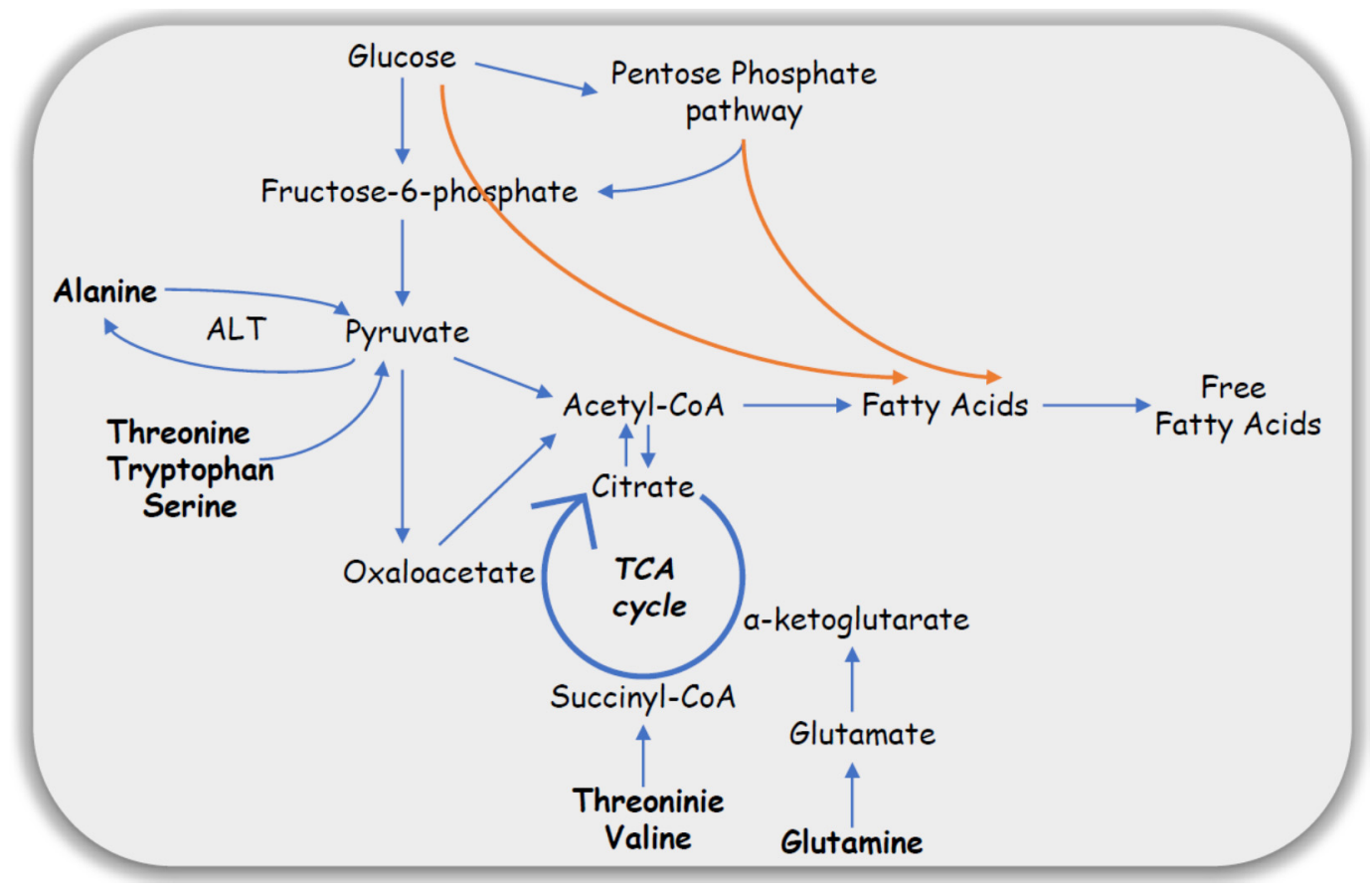

Figure 2. Alteration of glucose metabolism in PCOS.

In women with PCOS elevated levels of phenylalanine and glycated phenylalanine were also detected. The accumulation of the glycated AAs was also reported in T2DM [25]. The lower level of other AAs such as proline and histidine may be due to the increased utilization of these AAs as antioxidants during the oxidative stress present in PCOS.

Vitamin B6 metabolism may also be deranged in women with PCOS. One of its metabolic pathways is the synthesis and degradation of the AAs. The results of Chen et al. (2020) showed a significant increase of pyridoxal 5'-phosphate (PLP) and D-glutamic acid in the FF of women 
with PCOS. The authors claimed that both compounds are linked with the vitamin B6 metabolism. On the other hand, it is known that PLP is a coenzyme in the metabolism of homocysteine. Moreover, increased levels of homocysteine were observed in women with PCOS. Taking this into the account, disruption of the homocysteine metabolism in PCOS may impair the oocyte microenvironment. The second metabolite detected by the authors was glutamic acid. Its levels are strictly associated with efficiency of the glutamate decarboxylase, while the activity of this enzyme is regulated by the presence of vitamin B6. Glutamic acid is essential for the growth of oocytes, because it can be utilized as an alternative source of energy. These authors also suggested that the increased level of this metabolite is due to its accumulation in the FF [48].

Tang et al. (2019) pointed out that amino acid metabolic abnormalities are also characteristic of PCOS [51]. Among the branched-chain AAs, three were pointed out: valine, leucine, and isoleucine. All of them were up-regulated in women with PCOS. It is assumed that increased levels of valine, leucine, and isoleucine may affect the progression of the IR and obesity. From a biological point of view, branched-chain AAs can serve as substrates for the synthesis of glucose. It occurs in the case of IR, where abnormal glucose metabolism is carried out and alanine is obtained by the transamination of pyruvic acid. Second subgroup of AAs, which are associated with IR are lysine, phenylalanine, and 2-aminoadipic acid. Studies conducted by Tang et al. (2019) pointed to significantly higher concentrations of these metabolites in women with PCOS, which can also be associated with the IR [51]. Chen et al. (2020) reported that phenylalanine may be important for the growth and development of oocytes and could therefore be associated with ovulatory dysfunction [48].

IR plays a key role in the pathophysiology of PCOS and is associated with ovulatory dysfunction and hyperandrogenism. Some mechanisms of IR in women with PCOS are connected to an excessive activity of $17 \alpha$-hydroxylase, which regulates the conversion of 17-hydroxyprogesterone into androstenedione, excessive stimulation of IGF-I receptors, and diminished synthesis of insulin-like growth factor binding protein 1 (IGF-BP1) [52].

The Rotterdam diagnostic criteria yield four separate PCOS phenotypes (A, B, C, and D). Phenotype A includes all the three features (HA, AnO, and PCOM), whereas phenotype B, C, and D include only two (HA and AnO or HA and PCOM or AnO and PCOM, respectively). Among the mentioned PCOS studies, only one analyzed the metabolites among four different PCOS phenotypes (A, B, C, and D). Zhao et al. (2012) reported elevated levels of leucine and decreased levels of serine and threonine in women with the $\mathrm{C}(\mathrm{HA}+\mathrm{PCOM})$ phenotype in comparison to the other phenotypes [25].

\section{Limitations of Metabolomic Studies}

The range of described metabolites identified during metabolomic studies is enormous and gives an overview of the metabolic profile of women with PCOS. Several different compounds and many biochemical pathways seem to be involved in the pathogenesis of PCOS, which indicates the complexity of this common endocrinopathy. These alterations may be caused by an increased or reduced efficiency of different biochemical reactions, up- or down-regulation of genes, increased or decreased activity of enzymes, as well the formation of alternative metabolic pathways. Metabolomics enables us to study these biochemical pathways, which might be involved in the pathogenesis of PCOS. However, it is important to remember that there are some limitations of metabolomic studies. An enormous challenge in research, which is performed with the use of human matrices is inter-individual variability, especially in women, in whom the range of the detected metabolites could be correlated to different hormone levels during the menstrual cycle. Some metabolomic studies were performed relatively in a small group of women, with only tentatively identified metabolites. There could also be some difficulties with the efficiency of the analysers, which sometimes yield false positive results. Furthermore, differences in sample preparation also have a significant impact on the final results.

Therefore, in metabolomics research, every step of the study is significant-from appropriate patient requirements through analytical accuracy to the identification and statistical analysis of the 
obtained results. That is why there is the need to confirm if the detected compounds could become reliable biomarkers, which would selectively distinguish PCOS from other endocrinopathies.

\section{Conclusions}

Metabolomics has proven to be a potential tool in studying the pathophysiology of PCOS. The application of metabolomics allows us to discover metabolic pathways that have been shown to be deranged. These abnormalities are associated mainly with the metabolism of lipids, fatty acids, sphingolipids and glycerophospholipids, steroids as well as carbohydrates and amino acids (Figure 3). Additionally, some alternative biochemical processes have been shown to be up-regulated in women with PCOS; however, their clinical significance should be confirmed and evaluated. Determination of disturbed pathways allows identification of the specific compounds characteristic of PCOS, which might be considered as biomarkers and became potential targets for future metabolomic research. Finding appropriate biochemical markers could be a milestone in early diagnosis of this endocrinopathy and a starting point for targeted future pharmacological interventions.

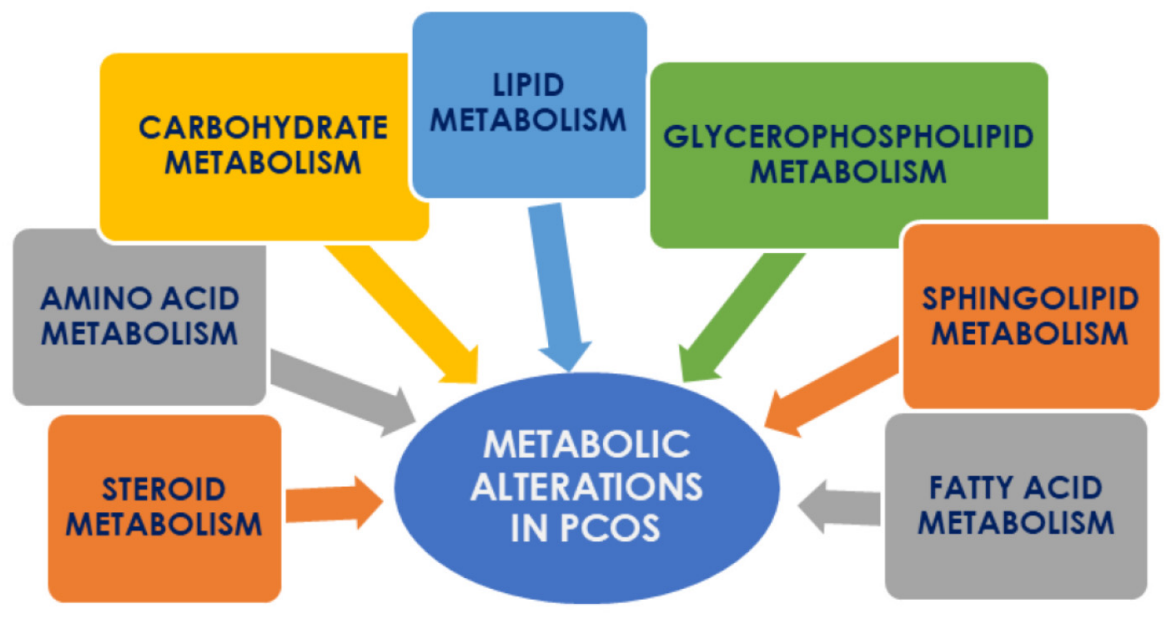

Figure 3. The main biochemical pathways disturbed in PCOS.

Author Contributions: Conceptualization, A.R.; resources, A.R.; data curation, A.R.; writing-original draft preparation, A.R. and M.B.-F.; writing-review and editing, M.B.-F., D.R.; visualization, A.R.; supervision, M.B.-F., D.R. and M.J.M.; funding acquisition, A.R. All authors have read and agreed to the published version of the manuscript.

Funding: This review article was financed by the Polish National Science Centre (grant number 2018/31/N/NZ7/03781).

Conflicts of Interest: The authors declare no conflict of interest.

\section{Abbreviations}

$\begin{array}{ll}\text { HA } & \text { Hyperandrogenism } \\ \text { AnO } & \text { Anovulatory oligomenorrhea } \\ \text { TV-US } & \text { Transvaginal ultrasound } \\ \text { PCOS } & \text { Polycystic ovary syndrome } \\ \text { PCOM } & \text { Polycystic ovary morphology } \\ \text { T2DM } & \text { Type 2 diabetes mellitus } \\ \text { AH } & \text { Arterial hypertension } \\ \text { MetS } & \text { Metabolic syndrome } \\ \text { NAFLD } & \text { Non-alcoholic fatty liver disease } \\ \text { CVD } & \text { Cardiovascular disease } \\ \text { IR } & \text { Insulin resistance }\end{array}$




$\begin{array}{ll}\text { HMDB } & \text { Human Metabolome Database } \\ \text { MS } & \text { Mass spectrometry } \\ \text { GC-MS } & \text { Gas chromatography-mass spectrometry } \\ \text { LC-MS } & \text { Liquid chromatography-mass spectrometry } \\ \text { NMR } & \text { Nuclear magnetic resonance } \\ \text { FF } & \text { Follicular fluid } \\ \text { PPAR } \gamma & \text { Peroxisome proliferator-activated receptor } \gamma \\ \text { PUFAs } & \text { Poly-unsaturated fatty acids } \\ \text { IGF-BP1 } & \text { Insulin-like growth factor binding protein } 1\end{array}$

\section{References}

1. Apridonidze, T.; Essah, P.A.; Iuorno, M.J.; Nestler, J.E. Prevalence and Characteristics of the Metabolic Syndrome in Women with Polycystic Ovary Syndrome. J. Clin. Endocrinol. Metab. 2005, 90, 1929-1935. [CrossRef]

2. Crespo, R.; Bachega, T.; Mendonça, B.; Gomes, L. An update of genetic basis of PCOS pathogenesis. Arch. Endocrinol. Metab. 2018, 62, 352-361. [CrossRef] [PubMed]

3. Rosenfiel, R.L. The Diagnosis of Polycystic Ovary Syndrome in Adolescents. Pediatr. Vol. 2015, 136, 1154-1165. [CrossRef]

4. Amiri, F.N.; Tehrani, F.R.; Simbar, M.; Montazeri, A.; Ali, R.; Thamtan, M. The Experience of Women Affected by Polycystic Ovary Syndrome: A Qualitative Study From Iran. Int. J. Endocrinol. Metab. 2014, 12, e13612.

5. Rocha, A.L.L.; Faria, L.C.; Guimarães, T.C.M.; Moreira, G.V.; Cândido, A.L.; Couto, C.A.; Reis, F.M. Non-alcoholic fatty liver disease in women with polycystic ovary syndrome: Systematic review and meta-analysis. J. Endocrinol. Investig. 2017, 40, 1279-1288. [CrossRef]

6. Carmina, E.; Bucchieri, S.; Esposito, A.; Del Puente, A.; Mansueto, P.; Orio, F.; Fede, G.D.; Rini, G. Abdominal Fat Quantity and Distribution in Women with Polycystic Ovary Syndrome and Extent of Its Relation to Insulin Resistance. J. Clin. Endocrinol. Metab. 2007, 92, 2500-2505. [CrossRef]

7. Shukla, P.; Mukherjee, S. Mitochondrial dysfunction: An emerging link in the pathophysiology of polycystic ovary syndrome. Mitochondrion 2020, 52, 24-39. [CrossRef]

8. Liu, Q.; Xie, Y.-J.; Qu, L.; Zhang, M.; Mo, Z. Dyslipidemia involvement in the development of polycystic ovary syndrome. Taiwan J. Obstet. Gynecol. 2019, 58, 447-453. [CrossRef] [PubMed]

9. Papadakis, G.; Kandarako, E.; Papalou, O.; Vryonidou, A.; Diamanti-Kandarakis, E. Is cardiovascular risk in women with PCOS a real risk? Current insights. Minerva Endocrinol. 2017, 42, 340-355.

10. Azziz, R.; Carmina, E.; Chen, Z.; Dunaif, A.; Laven, J.S.E.; Legro, R.S.; Lizneva, D.; Natterson-Horowtiz, B.; Teede, H.J.; Yildiz, B.O. Polycystic ovary syndrome. Nat. Rev. Dis. Prim. 2016, 2, 16057. [CrossRef]

11. Murri, M.; Insenser, M.; Escobar-Morreale, H.F. Metabolomics in polycystic ovary syndrome. Clin. Chim. Acta 2014, 429, 181-188. [CrossRef]

12. Yan, D.-K.; Liu, R.-H.; Jin, H.-Z.; Liu, X.-R.; Ye, J.; Shan, L.; Zhang, W.-D. “Omics” in pharmaceutical research: Overview, applications, challenges, and future perspectives. Chin. J. Nat. Med. 2015, 13, 3-21. [CrossRef]

13. Diamanti-Kandarakis, E.; Piperi, C.; Spina, J.; Argyrakopoulou, G.; Papanastasiou, L.; Bergiele, A.; Panidis, D. Polycystic Ovary Syndrome: The influence of environmental and genetic factors. Hormones 2006, 5, 17-34. [CrossRef] [PubMed]

14. Lindon, J.C.; Holmes, E.; Bollard, M.E.; Stanly, E.G.; Nicholson, J.K. Metabonomics technologies and their applications in physiological monitoring, drug safety assessment and disease diagnosis. Biomarkers 2004, 9, 1-31. [CrossRef]

15. Luque-Ramírez, M.; Millán, J.L.S.; Escobar-Morreale, H.F. Genomic variants in polycystic ovary syndrome. Clin. Chim. Acta 2006, 366, 14-26. [CrossRef] [PubMed]

16. Atiomo, W.; Khalid, S.; Parameshweran, S.; Houda, M.; Layfield, R. Proteomic biomarkers for the diagnosis and risk stratification of polycystic ovary syndrome: A systematic review. Int. J. Obstet. Gynaecol. 2009, 116, 137-143. [CrossRef] [PubMed]

17. Barderas, M.G.; Laborde, C.M.; Posada, M.; de la Cuesta, F.; Zubiri, I.; Vivanco, F.; Alvarez-Llamas, G. Metabolomic profiling for identification of novel potential biomarkers in cardiovascular diseases. J. Biomed. Biotechnol. 2011, 2011, 790132. [CrossRef] [PubMed] 
18. Lei, Z.; Huhman, D.V.; Sumner, L.W. Mass spectrometry strategies in metabolomics. J. Biol. Chem. 2011, 286, 25435-25442. [CrossRef]

19. Villas-Bôas, S.G.; Mas, S.; Åkesson, M.; Smedsgaard, J.; Nielsen, J. Mass spectrometry in metabolome analysis. Mass. Spectrom. Rev. 2005, 24, 613-646. [CrossRef]

20. Edison, A.S.; Markley, J.L.; Bru, R.; Eghbalnia, H.R.; Powers, R.; Raftery, D.; Wishart, D.S. The future of NMR-based metabolomics. Curr. Opin. Biotechnol. 2017, 43, 34-40.

21. Ma, X.; Fan, L.; Meng, Y.; Hou, Z.; Mao, Y.-D.; Wang, W.; Ding, W.; Liu, J.-Y. Proteomic analysis of human ovaries from normal and polycystic ovarian syndrome. Mol. Hum. Reprod. 2007, 13, 527-535. [CrossRef] [PubMed]

22. Matharoo-Ball, B.; Hughes, C.; Lancashire, L.; Tooth, D.; Ball, G.; Creaser, C.; Elgasim, M.; Rees, R.; Layfield, R.; Atiomo, W. Characterization of biomarkers in polycystic ovary syndrome (PCOS) using multiple distinct proteomic platforms. J. Proteome Res. 2007, 6, 3321-3328. [CrossRef]

23. Schmid, C.W. Metabolomics: What's happening downstream of DNA. Environ. Health Perspect. 2004, 112, 410-415.

24. Sun, L.; Hu, W.; Liu, Q.; Hao, Q.; Sun, B.; Zhang, Q.; Mao, S.; Qiao, J.; Yan, X. Metabonomics Reveals Plasma Metabolic Changes and Inflammatory Marker in Polycystic Ovary Syndrome Patients. J. Proteome Res. 2012, 11, 2937-2946. [CrossRef]

25. Zhao, Y.; Fu, L.; Li, R.; Wang, L.-N.; Yang, Y.; Liu, N.-N.; Zhang, C.-M.; Ying, W.; Ping, L.; Tu, B.-B.; et al. Metabolic profiles characterizing different phenotypes of polycystic ovary syndrome: Plasma metabolomics analysis. BMC Med. 2012, 10, 2012. [CrossRef] [PubMed]

26. Atiomo, W.; Daykin, C.A. Metabolomic biomarkers in women with polycystic ovary syndrome: A pilot study. Mol. Hum. Reprod. 2012, 18, 546-553. [CrossRef] [PubMed]

27. Escobar-Morreale, H.F.; Samino, S.; Insenser, M.; Vinaixa, M.; Luque-Ramírez, M.; Lasunción, M.A.; Correig, X. Metabolic heterogeneity in polycystic ovary syndrome is determined by obesity: Plasma metabolomic approach using GC-MS. Clin. Chem. 2012, 58, 999-1009. [CrossRef]

28. Whigham, L.D.; Butz, D.E.; Dashti, H.; Tonelli, M.; Johnson, L.K.; Cook, M.E.; Porter, W.P.; Eghbalnia, H.R.; Markley, J.L.; Steven, R.; et al. Metabolic Evidence of Diminished Lipid Oxidation in Women With Polycystic Ovary Syndrome. Curr. Metabolomics 2014, 2, 269-278. [CrossRef]

29. Zhao, X.; Xu, F.; Qi, B.; Hao, S.; Li, Y.; Zou, L.; Lu, C.; Xu, G.; Hou, L. Serum metabolomics study of polycystic ovary syndrome based on liquid chromatography-mass spectrometry. J. Proteome Res. 2014, 13, 1101-1111. [CrossRef]

30. Dong, F.; Deng, D.; Chen, H.; Cheng, W.; Li, Q.; Luo, R.; Ding, S. Serum metabolomics study of polycystic ovary syndrome based on UPLC-QTOF-MS coupled with a pattern recognition approach. Anal. Bioanal. Chem. 2015, 407, 4683-4695. [CrossRef]

31. RoyChoudhury, S.; Mishra, B.P.; Khan, T.; Chattopadhayay, R.; Lodh, I.; Datta Ray, C.; Bose, G.; Sarkar, H.S.; Srivastava, S.; Joshi, M.V.; et al. Serum metabolomics of Indian women with polycystic ovary syndrome using $1 \mathrm{H}$ NMR coupled with a pattern recognition approach. Mol. Biosyst. 2016, 12, 3407-3416. [CrossRef]

32. Szczuko, M.; Zapałowska-Chwyć, M.; Drozd, A.; Maciejewska, D.; Starczewski, A.; Stachowska, E. Metabolic pathways of oleic and palmitic acid are intensified in PCOS patients with normal androgen levels. Prostaglandins Leukot. Essent. Fat. Acids 2017, 126, 105-111. [CrossRef] [PubMed]

33. Buszewska-Forajta, M.; Rachoń, D.; Stefaniak, A.; Wawrzyniak, R. Identification of the metabolic fingerprints in women with polycystic ovary syndrome using the multiplatform metabolomics technique. J. Steroid Biochem. Mol. Biol. 2019, 186, 176-184. [CrossRef] [PubMed]

34. Jia, C.; Xu, H.; Xu, Y.; Xu, Y.; Shi, Q. Serum metabolomics analysis of patients with polycystic ovary syndrome by mass spectrometry. Mol. Reprod. Dev. 2019, 86, 292-297. [CrossRef] [PubMed]

35. Haoula, Z.; Ravipati, S.; Stekel, D.J.; Ortori, C.A.; Hodgman, C.; Daykin, C.; Raine-Fenning, N.; Barrett, D.A.; Atiomo, W. Lipidomic analysis of plasma samples from women with polycystic ovary syndrome. Metabolomics 2015, 11, 657-666. [CrossRef] [PubMed]

36. Fan, X.; Jiang, J.; Huang, Z.; Gong, J.; Wang, Y.; Xue, W.; Deng, Y.; Wang, Y.; Zheng, T.; Sun, A.; et al. UPLC/Q-TOF-MS based plasma metabolomics and clinical characteristics of polycystic ovarian syndrome. Mol. Med. Rep. 2019, 19, 280-292. [CrossRef] 
37. Vonica, C.L.; Ilie, I.R.; Socaciu, C.; Moraru, C.; Georgescu, B.; Farcaş, A.; Roman, G.; Mureşan, A.A.; Georgescu, C.E. Lipidomics biomarkers in women with polycystic ovary syndrome (PCOS) using ultra-high performance liquid chromatography-quadrupole time of flight electrospray in a positive ionization mode mass spectrometry. Scand. J. Clin. Lab. Investig. 2019, 79, 437-442. [CrossRef]

38. Fernández-Peralbo, M.A.; Luque de Castro, M.D. Preparation of urine samples prior to targeted or untargeted metabolomics mass-spectrometry analysis. Trends Anal. Chem. 2012, 41, 75-85. [CrossRef]

39. Wang, W.; Wang, S.; Tan, S.; Wen, M.; Qian, Y.; Zeng, X.; Guo, Y.; Yu, C. Detection of urine metabolites in polycystic ovary syndrome by UPLC triple-TOF-MS. Clin. Chim. Acta 2015, 448, 39-47. [CrossRef] [PubMed]

40. Zou, Y.; Zhu, F.-F.; Fang, C.-Y.; Xiong, X.-Y.; Li, H.-Y. Identification of Potential Biomarkers for Urine Metabolomics of Polycystic Ovary Syndrome Based on Gas Chromatography-Mass Spectrometry. Chin. Med. J. (Engl.) 2018, 131, 945-949. [CrossRef]

41. Dhayat, N.A.; Marti, N.; Kollmann, Z.; Troendle, A.; Bally, L.; Escher, G.; Grössl, M.; Ackermann, D.; Ponte, B.; Pruijm, M.; et al. Urinary steroid profiling in women hints at a diagnostic signature of the polycystic ovary syndrome: A pilot study considering neglected steroid metabolites. PLOS ONE 2018, 13, e0203903.

42. Arya, B.K.; Haq, A.U.; Chaudhury, K. Oocyte quality reflected by follicular fluid analysis in poly cystic ovary syndrome (PCOS): A hypothesis based on intermediates of energy metabolism. Med. Hypotheses 2012, 78, 475-478. [CrossRef] [PubMed]

43. McRae, C.; Sharma, V.; Fisher, J. Metabolite Profiling in the Pursuit of Biomarkers for IVF Outcome: The Case for Metabolomics Studies. Int. J. Reprod. Med. 2013, 2013, 1-16. [CrossRef] [PubMed]

44. Zhao, H.; Zhao, Y.; Li, T.; Li, M.; Li, J.; Li, R.; Liu, P.; Yu, Y.; Qiao, J. Metabolism alteration in follicular niche: The nexus among intermediary metabolism, mitochondrial function, and classic polycystic ovary syndrome. Free Radic. Biol. Med. 2015, 86, 295-307. [CrossRef]

45. Zhang, Y.; Liu, L.; Yin, T.; Yang, J.; Xiong, C.-L. Follicular metabolic changes and effects on oocyte quality in polycystic ovary syndrome patients. Oncotarget 2017, 8, 80472-80480. [CrossRef] [PubMed]

46. Liu, L.; Yin, T.-L.; Chen, Y.; Li, Y.; Yin, L.; Ding, J.; Yang, J.; Feng, H.L. Follicular dynamics of glycerophospholipid and sphingolipid metabolisms in polycystic ovary syndrome patients. J. Steroid Biochem. Mol. Biol. 2018, 185, 142-149. [CrossRef] [PubMed]

47. Sun, Z.; Chang, H.; Wang, A.; Song, J.; Zhang, X.; Guo, J.; Leung, P.C.K.; Lian, F. Identification of potential metabolic biomarkers of polycystic ovary syndrome in follicular fluid by SWATH mass spectrometry. Reprod. Biol. Endocrinol. 2019, 17, 45. [CrossRef]

48. Chen, X.; Lu, T.; Wang, X.; Sun, X.; Zhang, J.; Zhou, K.; Ji, X.; Sun, R.; Wang, X.; Chen, M.; et al. Metabolic alterations associated with polycystic ovary syndrome: A UPLC Q-Exactive based metabolomic study. Clin. Chim. Acta 2020, 502, 280-286. [CrossRef]

49. Tonks, K.T.; Coster, A.C.; Christopher, M.J.; Chaudhuri, R.; Xu, A.; Gagnon-Bartsch, J.; Chisholm, D.J.; James, D.E.; Meikle, P.J.; Greenfield, J.R.; et al. Skeletal muscle and plasma lipidomic signatures of insulin resistance and overweight/obesity in humans. Obesity 2016, 24, 908-916. [CrossRef]

50. Popovic, M.; Sartorius, G.; Christ-Crain, M. Chronic low-grade inflammation in polycystic ovary syndrome: Is there a (patho)-physiological role for interleukin-1? Semin. Immunopathol. 2019, 41, 447-459. [CrossRef]

51. Tang, L.; Yuan, L.; Yang, G.; Wang, F.; Fu, M.; Chen, M.; Liu, D. Changes in whole metabolites after exenatide treatment in overweight/obese polycystic ovary syndrome patients. Clin. Endocrinol. (Oxf.) 2019, 91, 508-516. [CrossRef] [PubMed]

52. Lewandowski, K.C.; Skowrońska-Jóźwiak, E.; Łukasiak, K.; Gałuszko, K.; Dukowicz, A.; Cedro, M.; Lewiński, A. How much insulin resistance in polycystic ovary syndrome? Comparison of HOMA-IR and insulin resistance (Belfiore) index models. Arch. Med. Sci. 2019, 15, 613-618. [CrossRef] [PubMed]

(C) 2020 by the authors. Licensee MDPI, Basel, Switzerland. This article is an open access article distributed under the terms and conditions of the Creative Commons Attribution (CC BY) license (http://creativecommons.org/licenses/by/4.0/). 